Number of pages: 16

Number of Figures: 13

Number of Tables: 8

Number of References: 28

\title{
Response of the seated human body to whole-body vertical vibration: Discomfort caused by sinusoidal vibration
}

\author{
Zhen Zhou and Michael J. Griffin \\ Human Factors Research Unit \\ Institute of Sound and Vibration Research \\ University of Southampton, \\ Southampton SO17 1BJ \\ England
}

Address for correspondence:

Professor Michael J Griffin

e-mail: M.J.Griffin@soton.ac.uk

Telephone: +44 (0)23 80592277

Facsimile: +44 (0)23 80592927 


\begin{abstract}
Frequency weightings for predicting vibration discomfort assume the same frequencydependence at all magnitudes of vibration, whereas biodynamic studies show the frequencydependence of the human body depends on the magnitude of vibration. This study investigated how the frequency-dependence of vibration discomfort depends on the acceleration and the force at the subject-seat interface. Using magnitude estimation, 20 males and 20 females judged their discomfort caused by sinusoidal vertical acceleration at 13 frequencies (1 to $16 \mathrm{~Hz}$ ) at magnitudes from 0.1 to $4.0 \mathrm{~ms}^{-2}$ r.m.s. The frequencydependence of their equivalent comfort contours depended on the magnitude of vibration, but was less dependent on the magnitude of dynamic force than the magnitude of acceleration, consistent with the biodynamic nonlinearity of the body causing some of the magnitude-dependence of equivalent comfort contours. There were significant associations between the biodynamic responses and subjective responses at all frequencies in the range 1 to $16 \mathrm{~Hz}$.
\end{abstract}

Keywords: ride comfort; frequency weighting; vibration magnitude; force.

\title{
Relevance of the findings for ergonomics practice
}

Vertical seat vibration causes discomfort in many forms of transport. This study provides the frequency dependence of vibration discomfort over a range of vibration magnitudes and shows how the frequency weightings in the current standards can be improved.

Running head: Discomfort caused by vibration 


\section{Introduction}

Human responses to whole-body vibration are dependent on many factors including the frequency of the vibration. From understanding of the frequency-dependence of the discomfort caused by vibration, frequency weightings have been developed, standardised (in British Standard 6841:1987, International Standard 2631-1:1997), and built into commercially available meters for measuring and evaluating exposures to whole-body vibration.

Understanding of the frequency-dependence of the discomfort caused by whole-body vibration is based on experimental studies that have determined the acceleration required to produce similar discomfort over a range of frequencies of vibration (i.e., equivalent comfort contours). Early studies asked subjects to estimate the extent of their sensations, discomfort, annoyance, etc. using semantic scales. More recent studies have mostly asked subjects to compare vibration stimuli so as to determine their relative importance in terms of some subjective response (e.g., Miwa 1967, Shoenberger and Harris 1971, Griffin et al. 1982, Corbridge and Griffin 1986, Howarth and Griffin 1988, Morioka and Griffin 2006a,b).

Equivalent comfort contours for the vertical vibration of seated people were obtained by Miwa (1967) using the method of adjustment, in which the discomfort caused by vibration at various frequencies in the range 0.5 to $300 \mathrm{~Hz}$ was compared with the discomfort caused by a reference vibration $(20 \mathrm{~Hz}$ at five different magnitudes: $0.01,0.0316,0.1$ and $0.316 \mathrm{~g}$, where $\mathrm{g}=9.81 \mathrm{~ms}^{-2}$ ). The equivalent comfort contours showed that subjects were most sensitive to vertical acceleration around 5 or $6 \mathrm{~Hz}$. Equivalent comfort contours over the frequency range 3.5 to $20 \mathrm{~Hz}$ (at 3.5, 5, 7, 9, 11, 15 and $20 \mathrm{~Hz}$ ) were obtained by Shoenberger and Harris (1971) using the method of magnitude estimation. With a $9-\mathrm{Hz}$ reference acceleration at $0.32 \mathrm{~g}$, it was found that subjects were most sensitive in the frequency range 5 to $10 \mathrm{~Hz}$. Using the method of constant stimuli, Griffin et al. (1982) obtained comfort contours equivalent to $0.8 \mathrm{~ms}^{-2}$ r.m.s. $10-\mathrm{Hz}$ vertical seat vibration from 36 subjects (18 males and 18 females) at the preferred one-third octave centre frequencies from 1 to $100 \mathrm{~Hz}$. It was found that the subjects were more sensitive to vertical vibration around $5 \mathrm{~Hz}$ and that male and female subjects produced similar equivalent comfort contours. Additional studies at octave centre frequencies from 1.0 to $63 \mathrm{~Hz}$ led to the conclusion that contours equivalent to 0.5 and $1.25 \mathrm{~ms}^{-2}$ r.m.s. had a broadly similar dependence on vibration frequency. Equivalent comfort contours for 40 subjects (20 males and 20 females) exposed to vertical sinusoidal vibration at the 11 preferred one-third octave centre frequencies from 0.5 to $5.0 \mathrm{~Hz}$ were obtained by Corbridge and Griffin (1986) relative to 0.25 and 0.75 $\mathrm{ms}^{-2}$ r.m.s. $2-\mathrm{Hz}$ vertical vibration. Subjects were more sensitive to acceleration at $5 \mathrm{~Hz}$ than to lower frequencies and, relative to their sensitivity at $2 \mathrm{~Hz}$, females were more sensitive than males to $5.0-\mathrm{Hz}$ vibration with the $0.25 \mathrm{~ms}^{-2}$ r.m.s. reference motion and to $3.15,4.0$ and $5.0 \mathrm{~Hz}$ with the $0.75 \mathrm{~ms}^{-2}$ r.m.s. reference motion. Equivalent comfort contours have also been expressed in terms of force (Mansfield and Maeda 2005): at 1, 2, and $4 \mathrm{~Hz}$ there was similar discomfort with similar force, whereas at frequencies greater than $4 \mathrm{~Hz}$, there was increasing sensitivity to force with increasing frequency (at 8,16 , and $31.5 \mathrm{~Hz}$ ). 
The experimentally determined equivalent comfort contours mentioned above provided the foundation for standardised frequency weightings for predicting the discomfort caused by acceleration measured at the subject-seat interface. British Standard 6841 (1987) advocates the use of frequency weighting $W_{\mathrm{b}}$ for the evaluation of vertical whole-body vibration. The asymptotic form of this weighting is independent of frequency ( $0 \mathrm{~dB}$ per octave) between 0.5 and $2 \mathrm{~Hz}$, increases in proportion to frequency ( $+6 \mathrm{~dB}$ per octave) between 2 and $5 \mathrm{~Hz}$, independent of frequency ( $0 \mathrm{~dB}$ octave) between 5 and $16 \mathrm{~Hz}$, and decreases inversely proportional to frequency ( $-6 \mathrm{~dB}$ per octave) between 16 and 80 $\mathrm{Hz}$, indicating greatest sensitivity to acceleration at frequency between 5 and $16 \mathrm{~Hz}$. International Standard 2631-1 (1997) uses a slightly modified frequency weighting, $W_{k}$, for the evaluation of some types of vertical vibration, based on a consensus among committee members rather than a 'consensus' among experimental findings.

The relation between the physical magnitude of a stimulus and the sensations it produces may be expressed by Stevens' power law, in which the 'objective magnitude', $\varphi$, of the stimulus and the 'subjective magnitude', $\psi$, of the response are assumed to be related by a power function:

$$
\Psi=\mathrm{k} \varphi^{\mathrm{n}}
$$

where the 'rate of growth of discomfort' with increasing magnitude of vibration is given by the exponent, $n$.

With fore-and-aft, lateral, and vertical vibration of both seated and standing people, the exponent, $n$, varies with the frequency of vibration (e.g., Morioka and Griffin 2006a, Wyllie and Griffin 2007, 2009, Thuong and Griffin 2011). The frequency-dependence of the exponent means that equivalent comfort contours have a different frequency-dependence at different magnitudes. Although this implies the frequency weightings should be different for low and high magnitude vibration this is not reflected in the frequency weightings in current standards.

The non-linearity in the weightings may be caused by several different phenomena. It seems likely that with some motions the neurophysiological mechanisms responsible for the sensations causing discomfort differ at low and high magnitudes of vibration. For example, while the sensations produced by low magnitudes of high frequency vibrotactile stimulation may be mediated by the Pacinian channel, higher magnitudes may be mediated by another tactile channel with a different frequencydependence. This may explain the magnitude-dependence of equivalent comfort contours seen in the exponents for hand-transmitted vibration (Morioka and Griffin 2006b) and high frequency whole-body vibration (Morioka and Griffin 2006a). With low frequency non-vertical vibration (e.g., roll or lateral oscillation), a change in the part of the body experiencing most discomfort as the magnitude of the oscillation increases may change the rate of increase in discomfort with increasing magnitude of oscillation (Wyllie and Griffin 2007).

Biodynamic studies have found that the mechanical responses of the body are also non-linear, with the resonance frequencies for whole-body vibration reducing as the magnitude of vibration increases. This nonlinearity has been seen with fore-and-aft, lateral, and vertical vibration of seated, standing and recumbent subjects (e.g., Fairley and Griffin 1989, Mansfield 1998, Matsumoto and Griffin 1998, 
Mansfield and Griffin 2000, Matsumoto and Griffin 2002a and 2002b, Matsumoto and Griffin 2005, Nawayseh and Griffin 2005, Subashi et al. 2006, 2009).

If the location in the body at which a vibration causes principal discomfort is distant from the location of the input (e.g., the supporting seat surface), the biodynamic non-linearity between the input and the location of discomfort may be expected to contribute to the frequency-dependence in the rate of growth of sensation, $n$. Matsumoto and Griffin (2005) found similar nonlinearities in the discomfort and the driving-point dynamic response associated with the principal body response to vertical vibration in the range 3.15 to $8 \mathrm{~Hz}$. They concluded that the nonlinearity in discomfort may be partially caused by the nonlinear dynamic response of the body and suggested the variation is sufficiently great to require consideration in methods of predicting the discomfort caused by vertical whole-body vibration. Subashi et al. (2009) found the magnitude of vibration had a similar effect on how vibration discomfort and apparent mass depend on the frequency of fore-and-aft and lateral whole-body vibration over the range 1.6 to $10 \mathrm{~Hz}$. There were significant correlations between discomfort and the normalised apparent mass.

When predicting vibration discomfort from the frequency-weighted acceleration at the supporting seat surface it is assumed that the same frequency weighting is appropriate at all vibration magnitudes. However, non-linearity in the response of the human body means that equivalent comfort contours have different frequency-dependence at different magnitudes and so the ideal frequency weighting is different for low magnitudes and high magnitude vibration. This study seeks to quantify the extent of the nonlinearity when predicting discomfort using acceleration at the seat surface. For the frequencies of vibration where discomfort arises from movements of body parts that have a large influence on the apparent mass of the body (i.e., frequencies up to and around the principal resonance of the body) it might be anticipated that the dynamic force would reflect the nonlinearity in vibration discomfort and provide a more accurate prediction of vibration discomfort.

This study was designed to determine equivalent comfort contours and the frequency-dependence of the rate of growth of discomfort for both acceleration and force measured at the surface supporting the seated human body. The apparent mass of the body was determined so as to quantify the biodynamic non-linearity and investigate the extent to which the frequency-dependence of the rate of change of discomfort could be attributed to the biodynamic nonlinearity. Assuming part of the nonlinearity in subjective responses is caused by biodynamic nonlinearity, it was hypothesised that equivalent comfort contours expressed in terms of dynamic force would show less nonlinearity than equivalent comfort contours expressed in terms of acceleration.

\section{Method}

\subsection{Apparatus}

A 1-metre stroke vertical electrohydraulic vibrator generated vertical vibration of a flat rigid seat. An accelerometer (Silicon Designs 2260-002) mounted on the lower surface of the seat measured acceleration in the direction of excitation. A force platform, Kistler 9281B, mounted on the seat measured the force at the interface between the seat (i.e., top surface of the force platform) and the 
subject in the vertical, fore-and-aft, and lateral directions. Sinusoidal vibration was generated by a Servotest Pulsar system and acquired using an HVLab data acquisition and analysis system (version 1.0) to a computer.

Subjects sat on the top surface of the seat without making contact with the backrest (Figure 1). They rested their feet on a footrest that was attached to the vibrator table.

\section{FIGURE 1 ABOUT HERE}

The distortions of the sinusoidal acceleration waveforms were examined by fitting measured waveforms to the desired waveforms. For all sinusoidal waveforms, the difference, $\delta_{a}$, between the measured and desired acceleration was calculated from:

$$
\delta_{a}=\frac{\int\left(a_{d}(t)-a_{m}(t)\right)^{2} d t}{\int\left(a_{d}(t)\right)^{2} d t} \times 100 \%
$$

where $a_{d}(t)$ is the desired acceleration, and $a_{m}(t)$ is the measured acceleration. For the session of the experiment using low magnitude stimuli, the median difference between the measured acceleration waveform and the desired acceleration waveform (i.e., ठa) was $1.7 \%$ (with a $5 \%-95 \%$ range from $0.43 \%$ to $5.5 \%$ ). The distortions were less with the greater magnitude stimuli employed in the sessions with medium and high magnitudes (see below).

\subsection{Subjects}

Twenty male and twenty female subjects, students and staff at the University of Southampton, participated in the study. Subject characteristics are shown in Table 1.

\section{TABLE 1 ABOUT HERE}

Subjects were exposed to white noise at $65 \mathrm{~dB}(\mathrm{~A})$ via a pair of headphones. During exposure to vibration, subjects were asked to close their eyes to prevent vision affecting their reaction to the motion.

The experiment was also designed to measure the nonlinearity in the vertical apparent mass and the fore-and-aft cross-axis apparent mass with both sinusoidal and random vibration. These biodynamic responses are reported separately (Zhou and Griffin, 2014). The experiment was approved by the Human Experimentation Safety and Ethics Committee of the Institute of Sound and Vibration Research at the University of Southampton. Informed consent to participate in the experiment was given by all subjects.

\subsection{Experiment design}

Subjects attended three sessions on three different days. In each session they were exposed to a series of sinusoidal vibrations presented in pairs: a 6-s 'reference' motion followed by a 6-s 'test' motion, with the first $1 \mathrm{~s}$ and last $1 \mathrm{~s}$ of both stimuli tapered by cosine functions. The two stimuli were separated by an interval of $1 \mathrm{~s}$.

The 'reference motion' had a frequency of $4 \mathrm{~Hz}$ and was presented at one of three magnitudes $(0.125$, 0.315 , or $0.8 \mathrm{~ms}^{-2}$ r.m.s.), with the magnitude constant within a session. The three sessions are referred 
to as the 'low magnitude session', 'medium magnitude session', and 'high magnitude session'. The 'test' motions were presented at each of 13 preferred one-third octave centre frequencies from 1.0 to $16 \mathrm{~Hz}$. At each frequency, the test motion was presented at nine magnitudes (equi-spaced on a logarithmic scale) that varied according to the frequency of the test motion (so as to produce an approximately similar range of subjective responses at each frequency, assuming the frequency-dependence of frequency weighting $W_{\mathrm{b}}$ ). The magnitudes of the test motion at $4 \mathrm{~Hz}$ (the frequency of the reference motion) varied from $40 \%$ of the magnitude of the reference motion to $250 \%$ of the magnitude of the reference motion (Table 2). In each session, subjects experienced 117 test motions in a completely randomised order over about 45 minutes.

\section{TABLE 2 ABOUT HERE}

Judgements of discomfort were obtained using the method of magnitude estimation, assuming the magnitude of discomfort caused by the reference motion was 100. For example, if a test motion was half as uncomfortable as the reference motion it should be assign a value of 50 , and a test motion twice as uncomfortable as the reference motion should be assigned a value of 200. If subjects could not feel a 'test' vibration, they were asked to give it a value of 0 , and their response was excluded from further analysis. Less than $2 \%$ of responses were excluded because subjects could not feel the lowest magnitude of vibration at some frequencies.

\subsection{Analysis}

For each frequency of vibration and each subject, the relation between the vibration acceleration, $\varphi$, and the individual magnitude estimate of discomfort, $\psi$, was determined using Stevens' Power law. Linear regression was performed at each frequency after logarithmic transformation of Equation (1) to:

$$
\log _{10} \psi=n \cdot \log _{10} \varphi+\log _{10} k
$$

The vertical apparent mass of each subject at each frequency was calculated by dividing the r.m.s. value of the measured force in vertical direction (after mass cancellation) by the r.m.s. value of the measured acceleration in the vertical direction:

$$
A M(f)=F_{\mathrm{rms}}(f) / A_{\mathrm{rms}}(f)
$$

where $A M(f)$ is the apparent mass at frequency $f$, and $F_{\text {rms }}(f)$ and $A_{\text {rms }}(f)$ are the r.m.s. values of the force and acceleration respectively. Mass cancellation was performed by subtracting the product of the mass of the top plate of the force platform and the vertical seat acceleration time history from the measured vertical force time history.

Statistical analysis was performed using non-parametric statistics. The Friedman two-way analysis of variance and the Wilcoxon matched-pairs signed ranks test were used to investigate differences between related samples and the Mann-Whitney U-test was to investigate differences between independent samples. Associations between variables were investigated with Spearman's rank correlation. The $p$-values shown are uncorrected for multiple comparisons. 


\section{Results}

3.1 Rate of growth of discomfort with increasing acceleration

The rate of growth of discomfort, $n$, and the constant, $k$, did not differ between male and female subjects at any of the 13 frequencies with any of the three magnitudes of the reference motion ( $p>0.05$, Mann-Whitney U-test). The median exponent, $n$, and the constant, $k$ over all 40 subjects are shown in Table 3. The medians and inter-quarter range of the rates of growth of discomfort, $n$, from all three magnitudes of the reference vibration are shown in Figure 2. With all three magnitudes of the reference vibration, the exponent, $n$, was highly dependent on the frequency of vibration $(p<0.0001$; Friedman). With all three magnitudes of the reference, at any frequency in the range 1 to $5 \mathrm{~Hz}$ the exponent was greater than at any frequency in the range 6.3 to $16 \mathrm{~Hz}(p<0.05$, Wilcoxon). At two frequencies, the exponent depended on the magnitude of the reference vibration $(p<0.003$ at 1.0 and 1.25, Friedman).

\section{FIGURE 2 AND TABLE 3 ABOUT HERE}

\subsection{Rate of growth of discomfort with increasing force}

For each vibration frequency and each subject, the relation between the measured force in the vertical direction, $\varphi$, and the individual sensation magnitude, $\psi$, was also determined using Stevens' Power law. The rate of growth of discomfort, $n$, and the constant $k$, did not differ between males and females at any of the 13 frequencies with any of the three magnitudes of the reference motion $(p>0.05$, Mann-Whitney U-test). The median exponent, $n$, and the constant, $k$, over all 40 subjects are shown in Table 4. The medians and inter-quarter range of the rates of growth of discomfort with increasing force are shown in Figure 3. With all three magnitudes of the reference motion, the rate of growth of discomfort, $n$, was highly dependent on the frequency of vibration ( $p<0.0001$; Friedman). Similar to acceleration, at two frequencies, the exponent depended on the magnitude of the reference vibration $(p<0.003$ at 1.0 and 1.25 , Friedman).

\section{FIGURE 3 AND TABLE 4 ABOUT HERE}

\subsection{Comparison of the rate of growth of discomfort between force and acceleration}

The rate of growth of discomfort, $n$, differed between force and acceleration at most frequencies with all three magnitudes of the reference motion (Table 5 and Figure 4). At frequencies less than $5 \mathrm{~Hz}$, the exponent for force was generally less than the exponent for acceleration, whereas at frequencies greater than $5 \mathrm{~Hz}$, the exponent for force was generally greater than the exponent for acceleration. Although the differences were small they were statistically significant at most frequencies. With the greatest magnitude of the reference motion, the difference was highly significant at all frequencies except $5 \mathrm{~Hz}(p<0.001$; Wilcoxon).

FIGURE 4 AND TABLE 5 ABOUT HERE 


\subsection{Equivalent comfort contours for acceleration}

Equivalent comfort contours for acceleration were determined for each subject by calculating the vibration acceleration, $\varphi$, corresponding to nine subjective magnitudes, $\psi$, from 40 to 250 at each vibration frequency (from 1 to $16 \mathrm{~Hz}$ ) using equation 1 . The equivalent comfort contours illustrate the vibration magnitudes required to produce the same strength of sensation across the frequency range. With each magnitude of the reference motion, the acceleration equivalent comfort contours for all sensation magnitudes varied with frequency $(p<0.001$, Friedman; Figure 5$)$.

\section{FIGURE 5 ABOUT HERE}

The acceleration equivalent comfort contours are roughly constant from 1 to $2 \mathrm{~Hz}$ and then reduce as the frequency increases. As the magnitude of vibration increased, the frequency at which the acceleration produced most discomfort decreased. The individual, median, and inter-quartile ranges of the equivalent comfort contours for acceleration (for $\psi=100$ ) are shown in Appendix 1.

Some of the contours in Figure 5 are lower than the lowest vibration magnitudes presented in a session of the study, especially for the lowest magnitudes at frequencies from 6.3 to $12.5 \mathrm{~Hz}$. However, the lowest 'extrapolated contours' with the $0.8 \mathrm{~ms}^{-2}$ r.m.s. reference are within the range of magnitudes studied with the $0.315 \mathrm{~ms}^{-2}$ r.m.s. reference. Similarly, the lowest 'extrapolated contours' with the $0.315 \mathrm{~ms}^{-2}$ r.m.s. reference are within the range of magnitudes studied with the $0.125 \mathrm{~ms}^{-2}$ r.m.s. reference. Overlapping these contours shows reasonable agreement, suggesting Stevens' power law can be used to make moderate extrapolations, at least at these higher magnitudes.

\subsection{Equivalent comfort contours for force}

Equivalent comfort contours for vertical force were determined for each subject by calculating the force, $\varphi$, corresponding to the same nine subjective magnitudes, $\psi$, from 40 to 250 at each vibration frequency (1 to $16 \mathrm{~Hz}$ ) using equation 1 . With all three magnitudes of the reference motion, the force equivalent comfort contours for all sensation magnitudes varied with frequency $(p<0.0001$, Friedman; Figure 6). Again, parts of some contours, especially for the lowest magnitudes at frequencies from 6.3 to $12.5 \mathrm{~Hz}$, are extrapolated to forces below the range of forces the subjects experienced.

\section{FIGURE 6 ABOUT HERE}

Similar to the acceleration equivalent comfort contours, the force equivalent comfort contours are roughly constant at low frequencies and reduce as the frequency increases. Subjects were most sensitive to force at $16 \mathrm{~Hz}$, except for a few of the lowest magnitudes of vibration. Comparing Figures 5 and 6 it can be seen that the force equivalent comfort contours are more parallel than the acceleration equivalent comfort contours, suggesting the force equivalent comfort contours are more 'linear'. The individual, median, and inter-quartile ranges of the equivalent comfort contours for force (for $\psi=100$ ) are shown in Appendix 2. 
3.6 Location of discomfort

Most discomfort was generally felt in either the buttocks or the upper body (Figure 7, Table 6). As the magnitude of vibration increased, the location of most discomfort moved from the lower-body to the upper-body. At most frequencies in the low magnitude session, there were some subjects who said they could not feel where the discomfort was located. In the middle frequency range, most subjects felt the discomfort in the buttocks or the upper-body during medium and high magnitude vibration.

\section{FIGURE 7 AND TABLE 6 ABOUT HERE}

\subsection{Association between relative discomfort and normalised apparent mass}

The association between subjective responses and biodynamic responses was investigated by calculating correlations between the ratio of apparent masses at two frequencies and the ratio of the subjective responses between the same two frequencies. The ratios were calculated for all possible pairs of frequencies for all subjects when exposed to the middle magnitude of vibration in each of the three sessions.

In the session with the greatest vibration magnitudes, there were no statistically significant negative correlations but distinct patterns of statistically significant positive correlations between the relative apparent mass and the relative subjective response (Table 7). For example, the ratio of the apparent mass at $4 \mathrm{~Hz}$ to the apparent mass at higher frequencies $(6.3 \mathrm{~Hz}$ to $16 \mathrm{~Hz})$ was positively correlated with the ratio of the subjective response at $4 \mathrm{~Hz}$ to the subjective response at these higher frequencies $(p<0.05$; Spearman). This indicates that subjects having a proportionately greater apparent mass at $4 \mathrm{~Hz}$ relative to their apparent mass at the higher frequencies were likely to be relatively more uncomfortable at $4 \mathrm{~Hz}$. Similarly, the ratio of the apparent mass at $6.3 \mathrm{~Hz}$ to the apparent mass at all lower frequencies $(1.0$ to $5.0 \mathrm{~Hz})$ was positively correlated with the ratio of the subjective response at $6.3 \mathrm{~Hz}$ to the subjective response at all lower frequencies ( $<<0.05$; Spearman). This indicates that subjects having a proportionately greater apparent mass at $6.3 \mathrm{~Hz}$ relative to their apparent mass at lower frequencies were likely to be relatively more uncomfortable at $6.3 \mathrm{~Hz}$. It can also be inferred that subjects having a proportionately greater apparent mass at $16 \mathrm{~Hz}$ relative to their apparent mass at frequencies less than $5 \mathrm{~Hz}$ were likely to be relatively more uncomfortable at $16 \mathrm{~Hz}$. In the sessions with medium vibration magnitudes the trends were similar, but the correlations with 4 $\mathrm{Hz}$ were only statistically significant at 8,10 , and $16 \mathrm{~Hz}$, the correlations with $6.3 \mathrm{~Hz}$ were only significant at 5 and $8 \mathrm{~Hz}$, and the correlations with $16 \mathrm{~Hz}$ were significant at $3.15,4,5$, and $12.5 \mathrm{~Hz}$. In the session with the lowest vibration magnitudes the correlations with $4 \mathrm{~Hz}$ were only statistically significant at 10, 12.5 and $16 \mathrm{~Hz}$, the correlations with $6.3 \mathrm{~Hz}$ were only significant at $1.0 \mathrm{~Hz}$, and the correlations with $16 \mathrm{~Hz}$ were only significant at 4 and $5 \mathrm{~Hz}$.

TABLE 7 ABOUT HERE 


\section{Discussion}

\subsection{Vibration discomfort for acceleration}

\subsubsection{Effect of acceleration magnitude}

The rate of growth for vertical vibration (i.e., the exponent in Stevens' power law) has been investigated previously for acceleration (Figure 8), but not for dynamic force. Over the preferred onethird octave centre frequencies between 2 and $315 \mathrm{~Hz}$ Morioka and Griffin (2006a) found the greatest rate of growth of discomfort for vertical vibration around the principal resonance frequency of the body, broadly consistent with this study. Similar values of the exponent were found at frequencies in the range 6.3 to $16 \mathrm{~Hz}$, but much greater values at lower frequencies in the present study, possibly because low frequency discomfort in the earlier study may have been influenced by relative motion between the seat and the stationary feet whereas there was no such relative motion in the present study (i.e., the feet and the seat moved with the same magnitude). Over the range of 4 to $63 \mathrm{~Hz}$ at magnitudes from 0.04 to $0.4 \mathrm{~ms}^{-2}$ r.m.s., Howarth and Griffin (1988) found no significant variation in the exponent with the frequency of vibration, unlike the present study with higher magnitudes of vibration where there were significant differences over the range 4 to $16 \mathrm{~Hz}$.

\section{FIGURE 8 ABOUT HERE}

Because the rate of growth of discomfort varied with the frequency of vibration, the shapes of the equivalent comfort contours varied with the magnitude of the vibration. A similar trend was observed with all three magnitudes of the reference vibration and for both male and female subjects, providing further evidence of nonlinearity in acceleration equivalent comfort contours. Shoenberger and Harris (1971) concluded that as the magnitude of a reference vibration increased, the frequency corresponding to the minimum of each acceleration contour decreased. Over the range 2 to 315 $\mathrm{Hz}$, Morioka and Griffin (2006a) found equivalent comfort contours similar to absolute perception thresholds with low magnitudes of acceleration (i.e., similar acceleration over the frequency range) but, with increasing sensation magnitudes, the contours changed to approximately similar velocity (i.e., acceleration increasing in proportion to frequency) over the frequency range 16 to $315 \mathrm{~Hz}$.

\subsubsection{Effect of frequency}

The median equivalent comfort contours (obtained for $\psi=100$ with each of the three magnitudes of the reference vibration) are compared in Figure 9. As the magnitude of the reference vibration increased, the frequency of acceleration producing greatest discomfort decreased. However, with all three magnitudes of vibration, greater discomfort was caused by acceleration at $5 \mathrm{~Hz}$ than acceleration at $4 \mathrm{~Hz}$.

\section{FIGURE 9 ABOUT HERE}

Greater sensitivity to vertical vibration acceleration at $5 \mathrm{~Hz}$ than at lower frequencies has been reported previously. This is apparent in contours equivalent to $0.75 \mathrm{~ms}^{-2}$ r.m.s. at $10 \mathrm{~Hz}$ obtained with 10 males at the nine preferred one-third octave centre frequencies from 3.15 to $20 \mathrm{~Hz}$ (Griffin 1976), in contours equivalent to $0.8 \mathrm{~ms}^{-2}$ r.m.s. at $10 \mathrm{~Hz}$ from 18 males and 18 females at preferred third- 
octave centre frequencies from 1 to $100 \mathrm{~Hz}$ (Griffin et al. 1982), in contours equivalent to 0.25 and $0.75 \mathrm{~ms}^{-2}$ r.m.s. with 20 males and 20 females over the 11 preferred one-third octave centre frequencies from 0.5 to $5.0 \mathrm{~Hz}$ (Corbridge and Griffin 1986), and in contours equivalent to $0.5 \mathrm{~ms}^{-2}$ r.m.s. $20 \mathrm{~Hz}$ vibration with 12 males over the 23 preferred one-third octave centre frequencies from 2 to $315 \mathrm{~Hz}$ (Morioka and Griffin 2006a).

The median acceleration equivalent comfort contours (for $\psi=100$ ) from the three sessions with the three different magnitudes of the reference vibration are compared with the findings of previous studies in Figure 10. With low frequency vibration, the equivalent comfort contours from the present study show similarities with the contours of Corbridge and Griffin (1986), where subjects also had no relative motion between the feet and the seat. The equivalent comfort contours from the present study also show close similarity to the contours of Griffin (1976) and Griffin et al. (1982) at frequencies greater than $3.15 \mathrm{~Hz}$ and Morioka and Griffin (2006a) at frequencies greater than about $5 \mathrm{~Hz}$. The subjects participating in these three studies had stationary feet and so relative motion between the seat and their feet may be expected to have increased subject discomfort at low frequencies (see Jang and Griffin 1999, 2000).

\section{FIGURE 10 ABOUT HERE}

The realisable forms of the $W_{\mathrm{b}}$ and $W_{\mathrm{k}}$ frequency weightings were inverted and normalised so that the accelerations at $4 \mathrm{~Hz}$ were the same as the reference vibration in each session (i.e., either 0.125 , 0.315 , or $0.8 \mathrm{~ms}^{-2}$ r.m.s.). The median equivalent comfort contours equivalent to the reference vibration (i.e., $\psi=100$ ) from each session were overlaid so that they could be compared with the inverted frequency weightings (Figure 11). With all three magnitudes of vibration, the $W_{\mathrm{b}}$ and $W_{\mathrm{k}}$ frequency weightings are broadly similar to the equivalent comfort contour at frequencies from 1 to 4 $\mathrm{Hz}$. The frequency weightings tend to underestimate discomfort from 4 to $16 \mathrm{~Hz}$ at all vibration magnitudes, but especially with lower magnitudes of vibration.

\section{FIGURE 11 ABOUT HERE}

Other studies also have questioned the applicability of currently standardised frequency weightings for the evaluation of low magnitude vibration (e.g., Morioka and Griffin 2006b).

\subsection{Vibration discomfort for force}

Similar to the equivalent comfort contours for acceleration, nonlinearity was also evident in the force equivalent comfort contours, although mostly with the lower magnitudes of vibration. The nonlinearity is less evident in the force contours in Figure 6 than in the acceleration contours in Figure 5, especially with greater magnitudes of vibration.

The dynamic force required to cause any degree of discomfort was almost constant at frequencies less than about $5 \mathrm{~Hz}$, but progressively decreased at frequencies greater than $5 \mathrm{~Hz}$ (Figure 6), consistent with the findings of Mansfield and Maeda (2005). It seems that less vertical force is required to cause discomfort as the frequency of vibration increases above the principal resonance in 
the vertical apparent mass of the seated human body around $5 \mathrm{~Hz}$. In this study, greatest sensitivity to vertical dynamic force occurred at the highest frequency studied (i.e., $16 \mathrm{~Hz}$ ). The head is the location where most discomfort was felt by seated subjects exposed to $16-\mathrm{Hz}$ vertical vibration (Figure 7 , and Whitham and Griffin 1978). Movement of the relatively low mass of the head requires less dynamic force than movement of the greater masses lower in the body that are responsible for discomfort at lower frequencies.

Current standards offer a six-point scale of discomfort (from 'not uncomfortable' to 'extremely uncomfortable') associated with various magnitudes of frequency-weighted acceleration (BS 6841, 1987; ISO 2631-1, 1997). Obviously, this scale should not be converted to a scale of force without recognising that forces are dependent on subject apparent mass, and therefore subject weight. Although force has some advantages over acceleration (e.g., less nonlinearity in the equivalent comfort contours) it cannot be used directly to predict discomfort without understanding the relation between subject mass and vibration discomfort. A doubling of dynamic force may approximately double vibration discomfort if it is associated with a doubling of acceleration, but probably not if it is associated with a doubling of subject mass.

\subsection{Gender}

The absence of significant differences in the acceleration comfort contours for males and females in the present study is reasonably consistent with the findings of previous studies. Similar contours were obtained from 18 male and 18 female subjects at one-third octave centre frequencies over the range 1 to $100 \mathrm{~Hz}$ (Griffin et al. 1982). With 20 males and 20 females providing contours equivalent to 0.25 and $0.75 \mathrm{~ms}^{-2}$ r.m.s. over the 11 preferred one-third octave centre frequencies from 0.5 to $5.0 \mathrm{~Hz}$, Corbridge and Griffin (1986) found females relatively more sensitive at 3.15, 4.0 and $5.0 \mathrm{~Hz}$ with a $0.75 \mathrm{~ms}^{-2}$ r.m.s. $2-\mathrm{Hz}$ reference motion and more sensitive at $5.0 \mathrm{~Hz}$ with a $0.25 \mathrm{~ms}^{-2}$ r.m.s. $2-\mathrm{Hz}$ reference motion.

The male subjects in the present study were significantly heavier than the female subjects $(p<0.0005$, Mann-Whitney U-test), so there were greater dynamic forces with the males, but these greater forces also occurred with the reference frequency, so were 'normalised'. Greater forces in heavier subjects might reasonably be expected to result in a greater risk of injury, but it is not clear whether they will also produce greater discomfort. The present study did not obtain absolute judgements of discomfort, merely judgements of discomfort relative to that caused by the reference frequency. The similarity in the equivalent comfort contours of the males and females is consistent with a similarity in the normalised apparent masses of these subjects (i.e., apparent mass divided by subject weight) as reported elsewhere (Zhou and Griffin 2014). Other studies have also found similar vertical apparent masses for males and females after normalisation (Fairley and Griffin, 1989, Toward and Griffin, 2011).

There were no significant differences in the rates of growth of discomfort, $n$, or the constant $k$, in Steven's Power law between males and females, so gender did not influence the shapes of the force equivalent comfort contours. 


\subsection{Frequency weightings}

The acceleration frequency weightings in current standards assume that the same frequencydependence is appropriate at all vibration magnitudes. In fact, the nonlinear response of human body means that equivalent comfort contours have a different frequency-dependence at different magnitudes and so the frequency weightings should differ at low and high vibration magnitudes. The equivalent comfort contours for force are less dependent on vibration magnitude, consistent with the biodynamic nonlinearities of the body (e.g., reductions in the resonance frequency with increasing vibration magnitude) contributing to the nonlinearity in the acceleration equivalent comfort contours. However, the nonlinearity evident in different rates of increase of discomfort with increasing vibration magnitude may also be due to differing sensitivity to changes in vibration magnitude in different parts of the body. Irrespective of the absolute threshold for perceiving vibration, the discomfort increases at a rate that depends on which part of the body produces the greatest sensation, which is dependent on the frequency of excitation and the magnitude of excitation. It seems likely that people find the vibration of some parts of their body more uncomfortable (or more unusual, or more concerning) than the vibration of other parts of their body and that the rate of growth of discomfort is greater when vibration excites these 'more sensitive' parts.

Consistent with various previous studies, the results show greater discomfort with acceleration at $5 \mathrm{~Hz}$ than acceleration at $4 \mathrm{~Hz}$, which is also consistent with the use of frequency weighting $W_{\mathrm{b}}$ for predicting vibration discomfort in preference to the use of frequency weighting $W_{\mathrm{k}}$ (see BS 6841:1987 and ISO 2631-1). This difference in sensitivity between $4 \mathrm{~Hz}$ and $5 \mathrm{~Hz}$ is relatively large (e.g., 65\% with the low magnitude vibration and $62 \%$ with the high magnitude vibration used in this study) and occurs at a frequency where seats often have resonances. The use of an inappropriate frequency weighting will prevent the optimisation of seat transmissibility.

The equivalent comfort contours for acceleration and force developed from this study can be constructed from the $n$ and $k$ values provided in Tables 4 and 5 and used to develop frequency weightings if required.

\subsection{Association between subjective and biodynamic response}

Associations between judgements of vibration discomfort and the apparent mass of the body have been found when investigating the nonlinearity of the body at low frequencies. Varying the magnitudes of whole-body vertical sinusoidal vibration and mechanical shocks, it was found that with increasing magnitude of excitation both the apparent mass normalised to $5 \mathrm{~Hz}$ and the discomfort normalised to $5 \mathrm{~Hz}$ increased at frequencies less than about $5 \mathrm{~Hz}$ (Matsumoto and Griffin 2005). In a linear system there would be no change in either the normalised discomfort or the normalised apparent mass when the magnitude of the excitation changes. This association between subjective and objective measures suggested the nonlinearity in the subjective response might be due to the nonlinearity in the apparent mass. A similar study investigated the relation between vibration discomfort and the apparent mass of the human body (both normalised at $4 \mathrm{~Hz}$ ) during fore-and-aft and lateral whole-body vibration (Subashi et al. 2009). With variations in the magnitude of the 
excitation, normalised judgements of vibration discomfort were again correlated with the normalised apparent mass at frequencies less than $5 \mathrm{~Hz}$. In both studies the correlations were less at higher frequencies, suggesting local motions in the body dominated discomfort at higher frequencies and did not greatly influence the forces measured at the seat and, therefore, the apparent mass. In a study of discomfort caused by whole-body vibration at frequencies over a wider range of frequencies (1 to 100 $\mathrm{Hz}$ ), it was concluded that increased seat-to-head transmissibility tended to be associated with increased subject discomfort (Griffin et al. 1982).

The present study adds additional evidence of the biodynamic responses of the body influencing vibration discomfort. For example, subjects having a proportionately greater apparent mass at $4 \mathrm{~Hz}$ were likely to be relatively more uncomfortable at $4 \mathrm{~Hz}$ and subjects having a proportionately greater apparent mass at $6.3 \mathrm{~Hz}$ were likely to be relatively more uncomfortable at $6.3 \mathrm{~Hz}$ (Table 7). Although the finding is unsurprising, it is helpful in giving confidence to the use of biodynamic measures to predict factors that will influence subjective responses. The present associations show that biodynamic differences between subjects (reflected in differences in the forces at the seat when the acceleration is the same) influence the frequency-dependence of discomfort caused by vertical vibration at frequencies over the range 1 to $16 \mathrm{~Hz}$.

\section{Conclusions}

With vertical whole-body vibration in the frequency range 1 to $16 \mathrm{~Hz}$, the frequency dependence of equivalent comfort contours, expressed in terms of either acceleration or force, vary with the magnitude of vibration. However, equivalent comfort contours for force show less variation with vibration magnitude, consistent with the biodynamic nonlinearity of the body contributing to the magnitude-dependence of equivalent comfort contours.

Over the range of magnitudes commonly encountered by seated passengers and operators, sensitivity to vertical acceleration is greater at $5 \mathrm{~Hz}$ than at lower frequencies, although this is not well reflected in the current International Standard.

Over the frequency range 1 to $16 \mathrm{~Hz}$, inter-subject differences in subjective responses to vertical vibration are associated with inter-subject variability in biodynamic responses.

\section{References}

British Standards Institution, BS 6841, 1987. Guide to measurement and evaluation of human exposure to whole-body mechanical vibration and repeated shock. London: British Standards Institution.

Corbridge, C. and Griffin, M.J., 1986. Vibration and comfort: Vertical and lateral motion in the range $0.5-5.0 \mathrm{~Hz}$. Ergonomics, 29, 249-272.

Fairley, T.E. and Griffin, M.J., 1989. The apparent mass of the seated human body: Vertical vibration. Journal of Biomechanics, 22 (2), 81-94.

Griffin, M.J., 1976. Subjective equivalence of sinusoidal and random whole-body vibration. Journal of Acoustical Society of America, 60 (5), 1140-1145.

Griffin, M.J., Whitham, E.M. and Parsons, K.C., 1982. Vibration and comfort. I: Translational seat vibration. Ergonomics, 25, 603-630.

Howarth, H.V.C. and Griffin, M.J., 1988. The frequency dependence of subjective reaction to vertical and horizontal whole-body vibration at low magnitudes. Journal of Acoustical Society of 
America, 83, 1406-1413.

International Organization of Standardization, ISO 2631-1, 1997. Mechanical vibration and shock evaluation of human exposure to whole-body vibration - part 1: General requirement. Geneva: ISO.

Jang, H.-K. and Griffin, M.J., 1999. The effect of phase of differential vertical vibration at the seat and feet on discomfort. Journal of Sound and Vibration, 223 (5), 785-794.

Jang, H.-K. and Griffin, M.J., 2000. Effect of phase, frequency, magnitude and posture on discomfort associated with differential vertical vibration at the seat and feet. Journal of Sound and Vibration, 229 (2), 273-286.

Mansfield, N.J. and Griffin, M.J., 1998. Effect of magnitude of vertical whole-body vibration on absorbed power for the seated human body. Journal of Sound and Vibration, 215 (4), 813825.

Mansfield, N.J. and Griffin, M.J., 2000. Non-linearities in apparent mass and transmissibility during exposure to whole-body vertical vibration. Journal of Biomechanics, 33 (8), 933-941.

Mansfield, N.J. and Maeda, S., 2005. Equal sensation curves for whole-body vibration expressed as a function of driving force. Journal of Acoustical Society of America, 117 (6), 3853-3859.

Matsumoto, Y. and Griffin, M.J., 1998. Dynamic responses of the standing human body exposed to vertical vibration: Influence of posture and vibration magnitude. Journal of Sound and Vibration, 212 (1), 85-107.

Matsumoto, Y. and Griffin, M.J., 2002a. Effect of muscle tension on non-linearities in the apparent masses of seated subjects exposed to vertical whole-body vibration. Journal of Sound and Vibration, 253 (1), 77-92.

Matsumoto, Y. and Griffin, M.J., 2002b. Non-linear characteristics in the dynamic responses of seated subjects exposed to vertical whole-body vibration. Journal of Biomechanical Engineering, 124 (5), 527-532.

Matsumoto, Y. and Griffin, M.J., 2005. Nonlinear subjective and biodynamic responses to continuous and transient whole-body vibration in the vertical direction. Journal of Sound and Vibration, 287, 919-937.

Miwa, T., 1967. Evaluation methods for vibration effect. Part1: Measurements of threshold and equal sensation contours of whole body for vertical and horizontal vibrations. Industrial Health, 5 , 183-205.

Morioka, M. and Griffin, M.J., 2006a. Magnitude-dependence of equivalent comfort contours for foreand-aft, lateral and vertical whole-body vibration. Journal of Sound and Vibration, 298 (3), 755-772

Morioka, M. and Griffin, M.J., 2006b. Magnitude dependence of equivalent comfort contours for fore and aft, lateral and vertical hand-transmitted vibration. Journal of Sound and Vibration, 295, 633-648.

Nawayseh, N. and Griffin, M.J., 2005. Non-linear dual-axis biodynamic response to fore-and-aft whole-body vibration. Journal of Sound and Vibration, 282, 831-862.

Shoenberger, R.W. and Harris, C.S., 1971. Psychophysical assessment of whole-body vibration. Human Factors, 13 (1), 41-50.

Subashi, G.H.M.J., Matsumoto, Y. and Griffin, M.J., 2006. Apparent mass and cross-axis apparent mass of standing subjects during exposure to vertical whole-body vibration. Journal of Sound and Vibration, 293, 78-95.

Subashi, G.H.M.J., Nawayseh, N., Matsumoto, Y. and Griffin, M.J., 2009. Nonlinear subjective and dynamic responses of seated subjects exposed to horizontal whole-body vibration. Journal of Sound and Vibration, 321 (1-2), 416-434.

Thuong, O. and Griffin, M.J., 2011. The vibration discomfort of standing persons: $0.5-16 \mathrm{~Hz}$ foreand-aft, lateral, and vertical vibration. Journal of Sound and Vibration 330, 816-826.

Toward, M. G.R., and Griffin, M J., 2011. Apparent mass of the human body in the vertical direction: Inter-subject variability. Journal of Sound and Vibration, 330, 827-841.

Wyllie, I.H. and Griffin, M.J., 2007. Discomfort from sinusoidal oscillation in the roll and lateral axes at frequencies between 0.2 and $1.6 \mathrm{~Hz}$. Journal of Acoustical Society of America, 121 (5), 26442654.

Wyllie, I.H. and Griffin, M.J., 2009. Discomfort from sinusoidal oscillation in the pitch and fore-and-aft axes at frequencies between 0.2 and $1.6 \mathrm{~Hz}$. Journal of Sound and Vibration, 324 (1-2), 453467.

Zhou, Z. and Griffin, M.J., 2014. Response of the seated human body to whole-body vertical vibration: Biodynamic responses to sinusoidal and random vibration. Ergonomics, submitted. 
Table 1 Subject characteristics: median (min-max)

\begin{tabular}{|l|l|l|l|l|}
\hline & Age (years) & Weight $(\mathrm{kg})$ & Standing height $(\mathrm{cm})$ & Sitting height $(\mathrm{cm})$ \\
\hline Male & $26.5(22-41)$ & $70.5(47.5-107)$ & $173(165-202)$ & $90(78-102)$ \\
\hline Female & $23.5(20-30)$ & $55.8(45-72)$ & $165(149-183)$ & $85.5(80-92)$ \\
\hline
\end{tabular}


Table 2 Magnitudes of test motions at each frequency in the three sessions with different magnitudes of the reference motion ( $\mathrm{ms}^{-2}$ r.m.s.)

\begin{tabular}{|c|c|c|c|c|c|c|}
\hline \multirow{3}{*}{$\begin{array}{c}\text { Frequency } \\
\text { of test } \\
\text { motion } \\
(\mathrm{Hz})\end{array}$} & \multicolumn{6}{|c|}{ Magnitude of $4-\mathrm{Hz}$ reference motion ( $\mathrm{ms}^{-2}$ r.m.s.) } \\
\hline & \multicolumn{2}{|c|}{$\begin{array}{c}\text { Low magnitude } \\
\left(0.125 \mathrm{~ms}^{-2} \text { r.m.s. }\right)\end{array}$} & \multicolumn{2}{|c|}{$\begin{array}{l}\text { Medium magnitude } \\
\left(0.315 \mathrm{~ms}^{-2} \text { r.m.s. }\right)\end{array}$} & \multicolumn{2}{|c|}{$\begin{array}{l}\text { High magnitude } \\
\left(0.8 \mathrm{~ms}^{-2} \text { r.m.s. }\right)\end{array}$} \\
\hline & Minimum & Maximum & Minimum & Maximum & Minimum & Maximum \\
\hline 1.0 & 0.10 & 0.63 & 0.25 & 1.6 & 0.63 & 4.0 \\
\hline 1.25 & 0.10 & 0.63 & 0.25 & 1.6 & 0.63 & 4.0 \\
\hline 1.6 & 0.10 & 0.63 & 0.25 & 1.6 & 0.63 & 4.0 \\
\hline 2.0 & 0.10 & 0.63 & 0.25 & 1.6 & 0.63 & 4.0 \\
\hline 2.5 & 0.08 & 0.50 & 0.20 & 1.25 & 0.5 & 3.2 \\
\hline 3.15 & 0.063 & 0.40 & 0.16 & 1.0 & 0.4 & 2.5 \\
\hline 4.0 & 0.05 & 0.315 & 0.125 & 0.8 & 0.315 & 2.0 \\
\hline 5.0 & 0.04 & 0.25 & 0.10 & 0.63 & 0.25 & 1.6 \\
\hline 6.3 & 0.04 & 0.25 & 0.10 & 0.63 & 0.25 & 1.6 \\
\hline 8.0 & 0.04 & 0.25 & 0.10 & 0.63 & 0.25 & 1.6 \\
\hline 10.0 & 0.04 & 0.25 & 0.10 & 0.63 & 0.25 & 1.6 \\
\hline 12.5 & 0.04 & 0.25 & 0.10 & 0.63 & 0.25 & 1.6 \\
\hline 16.0 & 0.04 & 0.25 & 0.10 & 0.63 & 0.25 & 1.6 \\
\hline
\end{tabular}


Table 3 Median exponent, $n$, and constant, $k$, for acceleration (in $\mathrm{ms}^{-2}$ r.m.s.) in each session (Low: 0.125 ms $^{-2}$ r.m.s. 4-Hz reference; Medium: $0.315 \mathrm{~ms}^{-2}$ r.m.s. 4-Hz reference; High: $0.8 \mathrm{~ms}^{-2}$ r.m.s. 4-Hz reference).

\begin{tabular}{|c|c|c|c|c|c|c|}
\hline \multirow{2}{*}{$\begin{array}{c}\text { Frequency } \\
(\mathrm{Hz})\end{array}$} & \multicolumn{3}{|c|}{ Exponent $(n)$} & \multicolumn{3}{c|}{ Constant $(k)$} \\
\cline { 2 - 7 } & Low & Medium & High & Low & Medium & High \\
\hline 1 & 1.05 & 0.95 & 1.29 & 370 & 194 & 65 \\
\hline 1.25 & 1.13 & 1.04 & 1.25 & 374 & 177 & 59 \\
\hline 1.6 & 0.93 & 1.09 & 1.17 & 433 & 172 & 64 \\
\hline 2 & 1.15 & 1.09 & 1.13 & 460 & 167 & 71 \\
\hline 2.5 & 1.25 & 1.04 & 1.18 & 561 & 211 & 80 \\
\hline 3.15 & 1.25 & 1.21 & 1.36 & 655 & 291 & 96 \\
\hline 4 & 1.18 & 1.18 & 1.14 & 763 & 363 & 135 \\
\hline 5 & 0.95 & 1.09 & 0.91 & 892 & 542 & 192 \\
\hline 6.3 & 0.73 & 0.61 & 0.73 & 783 & 364 & 179 \\
\hline 8 & 0.62 & 0.52 & 0.71 & 604 & 330 & 169 \\
\hline 10 & 0.59 & 0.64 & 0.75 & 573 & 368 & 164 \\
\hline 12.5 & 0.71 & 0.66 & 0.77 & 693 & 333 & 163 \\
\hline 16 & 0.82 & 0.64 & 0.71 & 809 & 358 & 159 \\
\hline
\end{tabular}


Table 4 Median exponent, $n$, and constant, $k$, for force (in Newtons) in each session (Low: $0.125 \mathrm{~ms}^{-2}$ r.m.s. 4-Hz reference; Medium: $0.315 \mathrm{~ms}^{-2}$ r.m.s. $4-\mathrm{Hz}$ reference; High: $0.8 \mathrm{~ms}^{-2}$ r.m.s. $4-\mathrm{Hz}$ reference).

\begin{tabular}{|c|c|c|c|c|c|c|}
\hline \multirow{2}{*}{$\begin{array}{c}\text { Frequency } \\
(\mathrm{Hz})\end{array}$} & \multicolumn{3}{|c|}{ Exponent $(n)$} & \multicolumn{3}{c|}{ Constant $(k)$} \\
\cline { 2 - 7 } & Low & Medium & High & Low & Medium & High \\
\hline 1 & 1.06 & 0.95 & 1.26 & 6.53 & 3.55 & 0.47 \\
\hline 1.25 & 1.12 & 1.02 & 1.24 & 3.68 & 2.34 & 0.47 \\
\hline 1.6 & 0.93 & 1.09 & 1.13 & 6.24 & 1.73 & 0.60 \\
\hline 2 & 1.15 & 1.08 & 1.08 & 4.39 & 2.16 & 1.04 \\
\hline 2.5 & 1.29 & 1.03 & 1.11 & 3.11 & 3.04 & 0.77 \\
\hline 3.15 & 1.26 & 1.20 & 1.24 & 4.66 & 1.87 & 0.55 \\
\hline 4 & 1.20 & 1.14 & 0.99 & 6.24 & 2.73 & 1.88 \\
\hline 5 & 0.97 & 1.10 & 0.94 & 11.66 & 5.09 & 3.38 \\
\hline 6.3 & 0.76 & 0.75 & 0.92 & 31.98 & 20.96 & 4.44 \\
\hline 8 & 0.70 & 0.62 & 0.80 & 34.67 & 30.01 & 8.70 \\
\hline 10 & 0.58 & 0.70 & 0.83 & 58.99 & 29.89 & 10.36 \\
\hline 12.5 & 0.80 & 0.70 & 0.89 & 50.00 & 28.98 & 9.68 \\
\hline 16 & 0.88 & 0.70 & 0.82 & 37.85 & 33.25 & 12.63 \\
\hline
\end{tabular}


Table 5 Statistical significance of differences in the rate of growth of discomfort, $n$, between force and acceleration ( $p$ value; Wilcoxon matched-pairs signed ranks test). (Low: $0.125 \mathrm{~ms}^{-2}$ r.m.s. 4-Hz reference; Medium: $0.315 \mathrm{~ms}^{-2}$ r.m.s. 4-Hz reference; High: $0.8 \mathrm{~ms}^{-2}$ r.m.s. 4-Hz reference).

\begin{tabular}{|c|c|c|c|c|c|c|c|c|c|c|c|c|c|}
\hline \multirow[b]{2}{*}{ Magnitude } & \multicolumn{13}{|c|}{ Frequency $(\mathrm{Hz})$} \\
\hline & 1.0 & 1.25 & 1.6 & 2.0 & 2.5 & 3.15 & 4.0 & 5.0 & 6.3 & 8.0 & 10 & 12.5 & 16 \\
\hline Low & - & - & - & ** & * & - & - & - & *夫 & $\star \star \star$ & - & 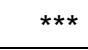 & $\star \star \star$ \\
\hline Medium & $\star \star \star$ & * & - & - & - & $\star \star \star$ & $\star \star \star$ & - & $\star \star \star$ & $\star \star \star$ & ** & *** & $\star \star \star$ \\
\hline High & $\star \star \star$ & $\star \star \star$ & 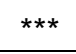 & $\star \star \star$ & $\star \star \star$ & $\star \star \star *$ & $\star \star \star *$ & * & $\star \star \star ~$ & $\star \star \star$ & $\star \star \star *$ & *** & $\star \star \star$ \\
\hline
\end{tabular}

${ }^{* * *} p<0.0005,{ }^{* *} p<0.005,{ }^{*} p<0.05,-$ not significant 
Table 6 Locations in the body where most subjects felt discomfort. (Low: $0.125 \mathrm{~ms}^{-2}$ r.m.s. 4-Hz reference; Medium: 0.315 ms ${ }^{-2}$ r.m.s. $4-\mathrm{Hz}$ reference; High: $0.8 \mathrm{~ms}^{-2}$ r.m.s. $4-\mathrm{Hz}$ reference).

\begin{tabular}{|c|c|c|c|c|c|c|c|c|c|c|c|c|c|c|}
\hline & $\begin{array}{l}\text { Frequency } \\
(\mathrm{Hz})\end{array}$ & 1.0 & 1.25 & 1.6 & 2.0 & 2.5 & 3.15 & 4.0 & 5.0 & 6.3 & 8.0 & 10 & 12.5 & 16 \\
\hline Low & Females & 1 & 1 & 1 & 1 & 1 & 1 & 1 & 4 & 5 & 5 & 4 & 4 & 2 \\
\hline Medium & Females & 9 & 9 & 7 & 5 & 5 & 4 & 5 & 5 & 7 & 7 & 4 & 3 & 4 \\
\hline \multirow{2}{*}{ High } & Males & 5 & 6 & 7 & 5 & 5 & 5 & 7 & 5 & 7 & 7 & 4 & 4 & 4 \\
\hline & Females & 6 & 5 & 5 & 7 & 7 & 7 & 7 & 7 & 7 & 4 & 9 & 9 & 9 \\
\hline
\end{tabular}

1: no specific location; 2: feet; 3: lower legs; 4: thighs; 5: buttocks; 6: lower body; 7: upper body; 8: neck; 9: head 
Table 7 Spearman correlation coefficients between the ratio of apparent mass at two frequencies and the ratio of the subjective responses between the same two frequencies (high magnitude session: $0.8 \mathrm{~ms}^{-2}$ r.m.s. $4-\mathrm{Hz}$ reference).

\begin{tabular}{|c|c|c|c|c|c|c|c|c|c|c|c|c|c|}
\hline $\begin{array}{l}\text { Frequency } \\
(\mathrm{Hz})\end{array}$ & 1 & 1.25 & 1.6 & 2 & 2.5 & 3.15 & 4 & 5 & 6.3 & 8 & 10 & 12.5 & 16 \\
\hline 1 & -- & -0.04 & -0.02 & -0.07 & 0.03 & 0.22 & 0.15 & -0.02 & $0.32^{*}$ & 0.13 & -0.05 & 0.23 & 0.30 \\
\hline 1.25 & & -- & 0.15 & 0.06 & -0.11 & 0.22 & 0.22 & -0.07 & $0.55^{\star \star \star}$ & 0.23 & -0.07 & 0.21 & $0.35^{\star}$ \\
\hline 1.6 & & & -- & -0.13 & -0.05 & 0.15 & 0.30 & -0.03 & $0.51^{\star \star *}$ & 0.30 & 0.15 & 0.14 & 0.31 \\
\hline 2 & & & & -- & -0.17 & 0.06 & 0.12 & 0.22 & $0.48^{\star \star \star}$ & $0.49 * *$ & $0.33^{*}$ & 0.21 & $0.44^{\star *}$ \\
\hline 2.5 & & & & & -- & -0.01 & 0.20 & -0.12 & $0.44^{* *}$ & $0.43^{\star \star}$ & 0.22 & 0.14 & $0.40^{*}$ \\
\hline 3.15 & & & & & & -- & 0.17 & 0.05 & $0.51^{\star \star \star}$ & $0.49 * \star$ & 0.30 & $0.36^{*}$ & $0.59 * \star \star$ \\
\hline 4 & & & & & & & -- & 0.22 & $0.71^{\star \star \star}$ & $0.60^{\star \star *}$ & $0.38^{\star}$ & $0.32^{*}$ & $0.58^{\star \star \star}$ \\
\hline 5 & & & & & & & & -- & $0.46^{\star *}$ & 0.18 & -0.02 & 0.12 & 0.19 \\
\hline 6.3 & & & & & & & & & -- & 0.13 & 0.17 & 0.10 & 0.09 \\
\hline 8 & & & & & & & & & & -- & -0.14 & -0.16 & -0.09 \\
\hline 10 & & & & & & & & & & & -- & -0.07 & 0.11 \\
\hline 12.5 & & & & & & & & & & & & -- & -0.07 \\
\hline 16 & & & & & & & & & & & & & -- \\
\hline
\end{tabular}

*: $p<0.05 ;{ }^{\star *}: p<0.01 ;{ }^{* \star *}: p<0.001$ 
Response of the seated human body to whole-body vertical vibration: discomfort caused by sinusoidal vibration Zhou, Z. \& Griffin, M. J. 14 Apr 2014 In : Ergonomics. 57, 5, p. 693-713

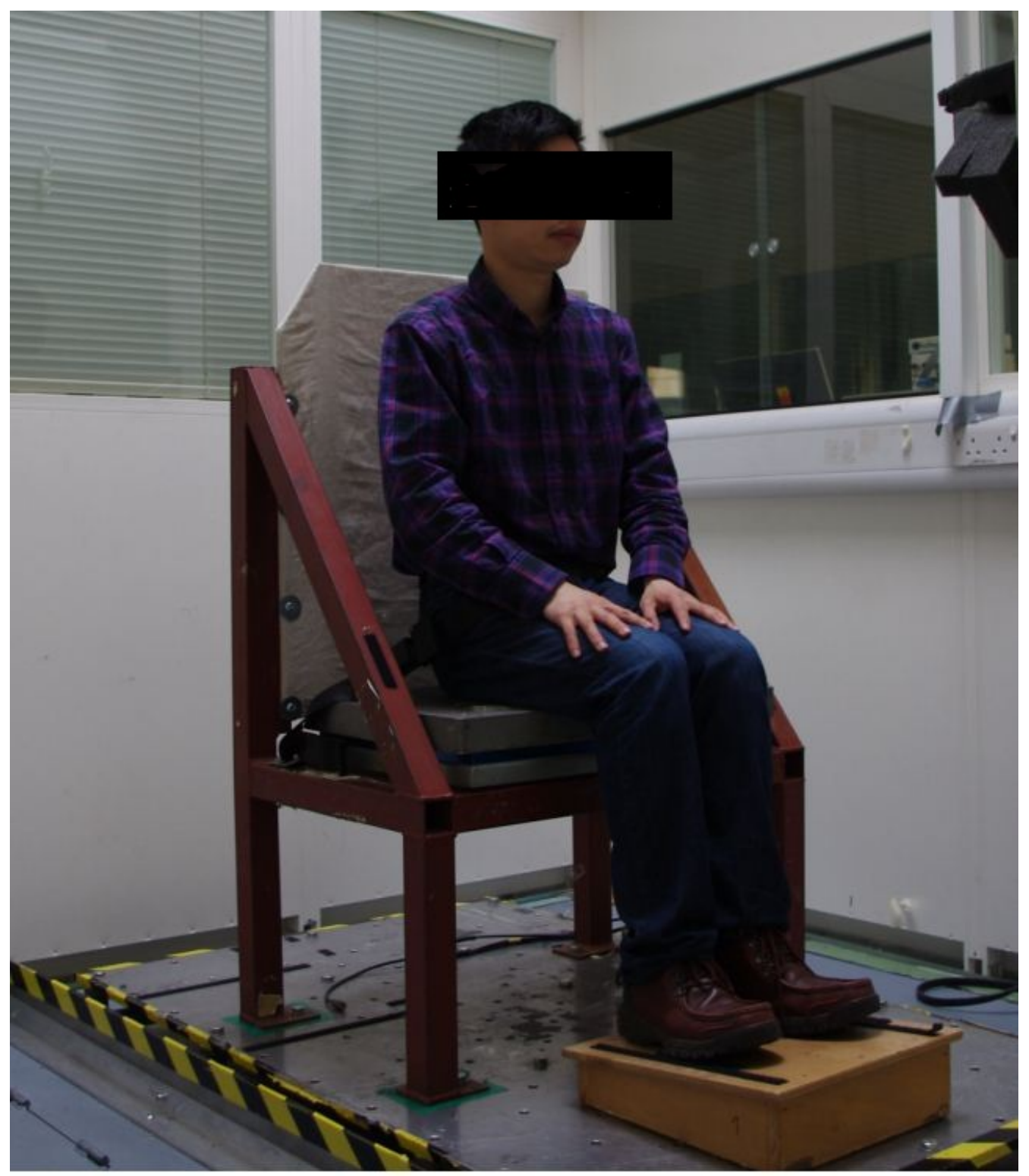

Figure 1 Experiment setup. 


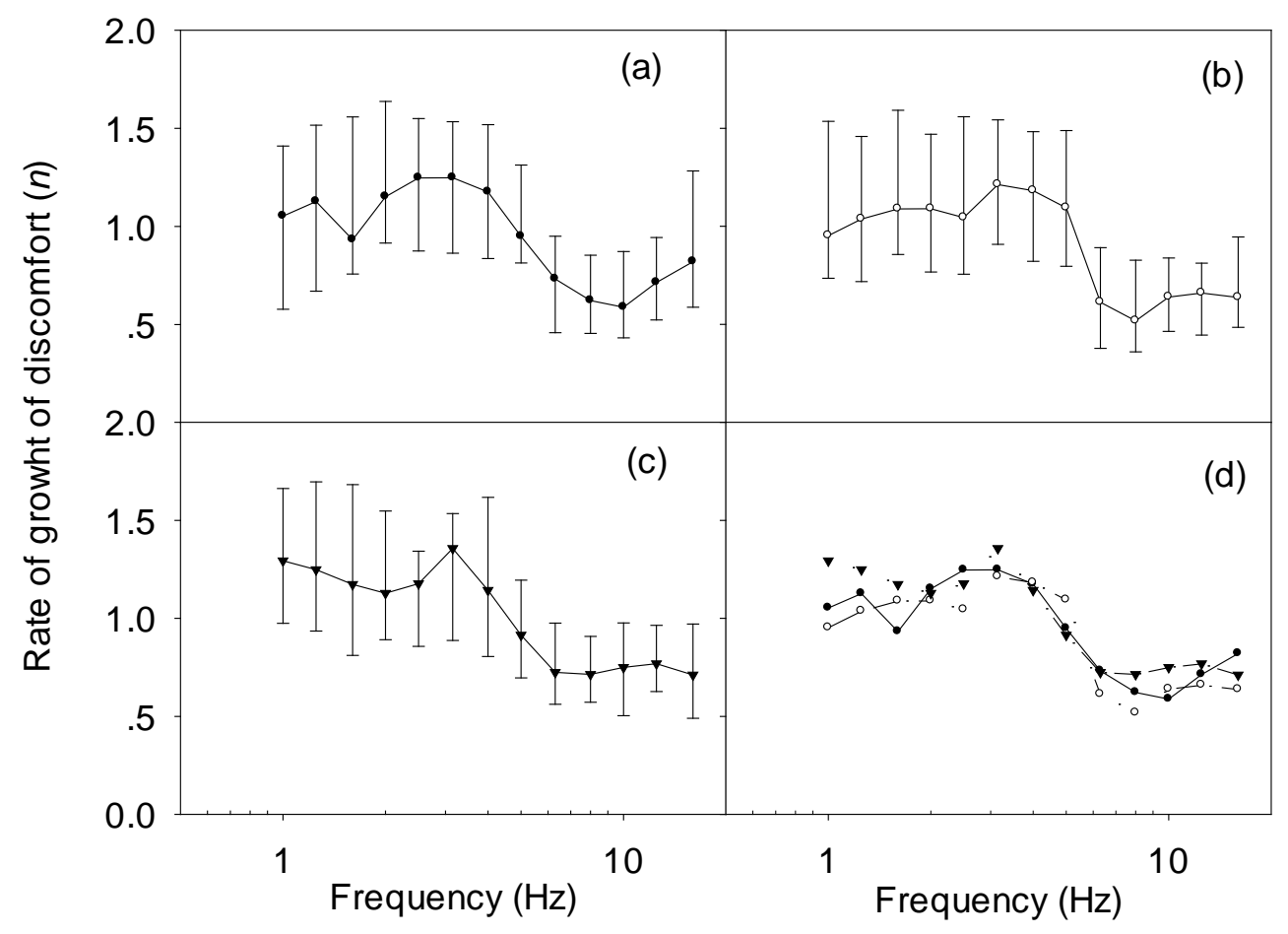

Figure 2

Median and inter-quartile range of rate of growth of discomfort, $n$, for vertical vibration acceleration with three magnitudes of $4-\mathrm{Hz}$ reference vibration. (a) Low magnitude session; (b) Medium magnitude session; (c) High magnitude session; (d) Median data for all three sessions, ๑: low magnitude (0.125 $\mathrm{ms}^{-2}$ r.m.s. 4-Hz reference); $\bigcirc$ : medium magnitude (0.315 $\mathrm{ms}^{-2}$ r.m.s. 4-Hz reference); $\boldsymbol{\nabla}$ : high magnitude (0.8 $\mathrm{ms}^{-2}$ r.m.s. 4-Hz reference). 


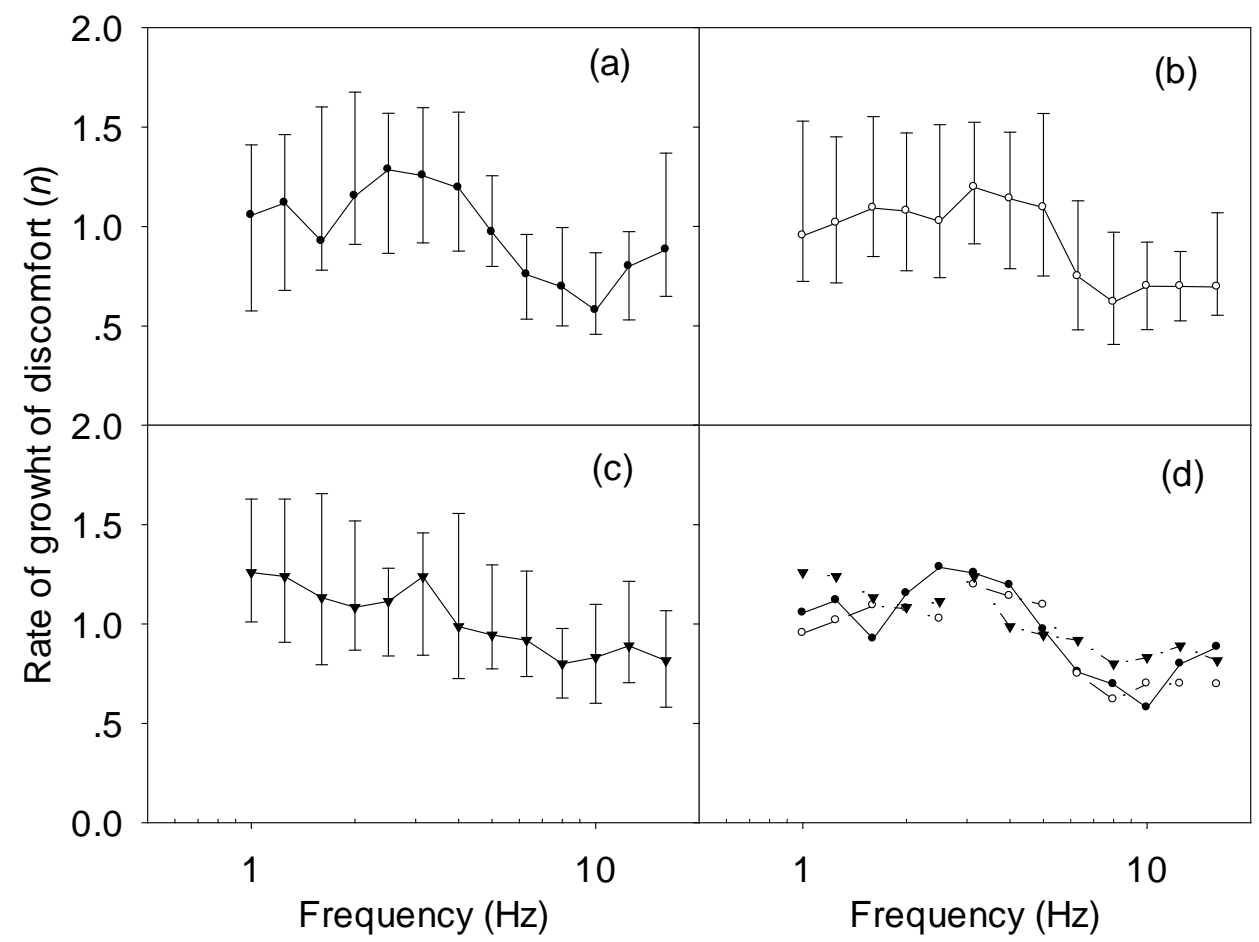

Figure 3

Median and inter-quartile range rate of growth of discomfort, $n$, for vertical vibration force with three magnitudes of $4-\mathrm{Hz}$ reference vibration. (a) Low magnitude session; (b) Medium magnitude session; (c) High magnitude session; (d) Median data for all three sessions, low magnitude; $\bigcirc$ : medium magnitude; $\nabla$ : high magnitude. 


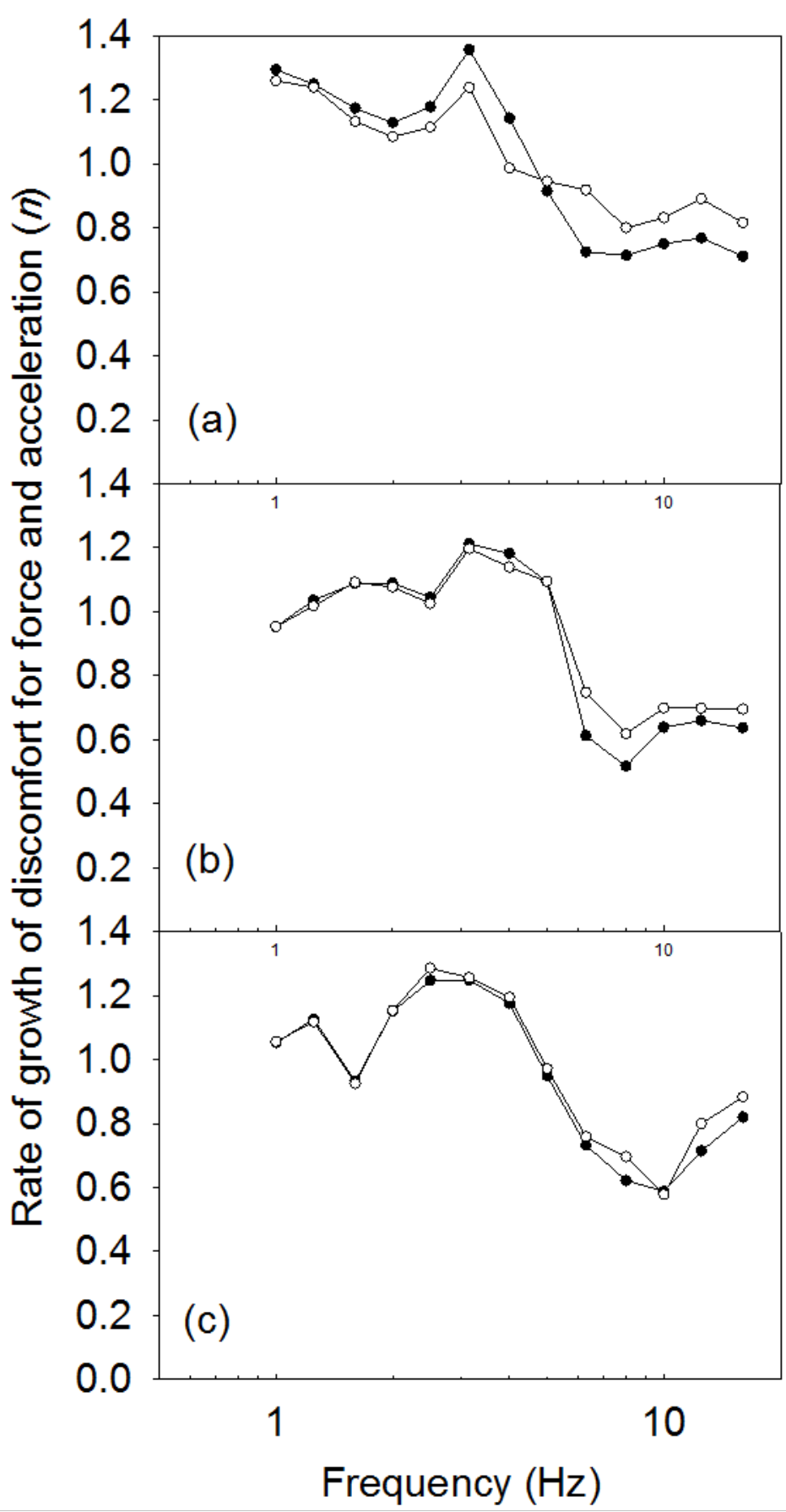

Figure 4 Comparison of the rates of growth of discomfort for force and acceleration obtained with three magnitudes of $4-\mathrm{Hz}$ reference vibration. Median data: $\bullet$ : acceleration; $\circ$ : force. (a): high magnitude (0.8 $\mathrm{ms}^{-2}$ r.m.s.); (b) medium magnitude (0.315 $\mathrm{ms}^{-2}$ r.m.s.); (c) low magnitude (0.125 $\mathrm{ms}^{-2}$ r.m.s.). 


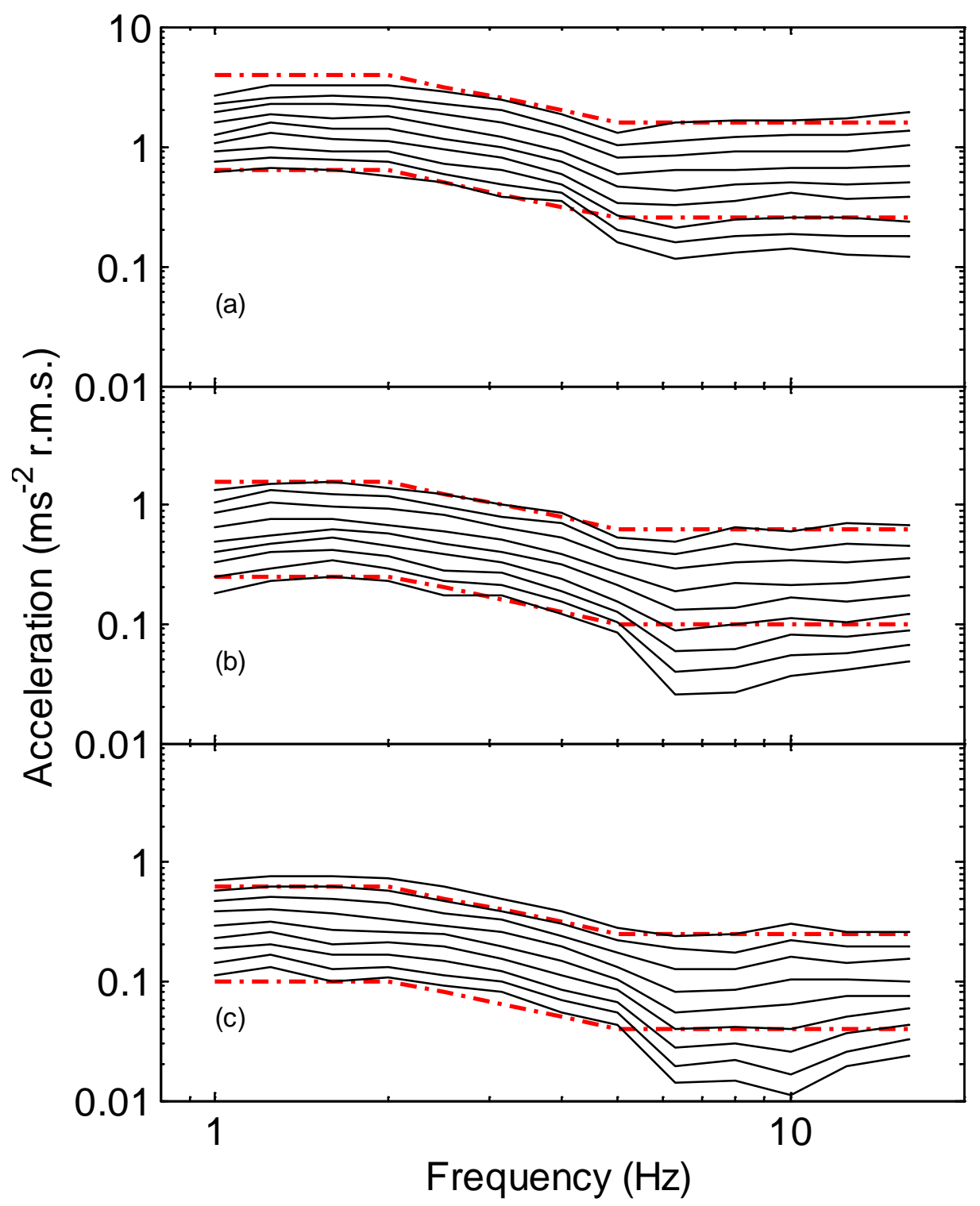

Figure 5

Median acceleration equivalent comfort contours. Contours are shown for subjective magnitudes, $\psi$, of 40,50,63, 80, $100125,160,200$ and 250 with three magnitudes of $4-\mathrm{Hz}$ reference vibration. (a): high magnitude $\left(0.8 \mathrm{~ms}^{-2}\right.$ r.m.s.); (b) medium magnitude $\left(0.315 \mathrm{~ms}^{-2}\right.$ r.m.s.); (c) low magnitude $\left(0.125 \mathrm{~ms}^{-2}\right.$ r.m.s.). The red broken lines show the ranges of magnitudes of the test motions in each session. 


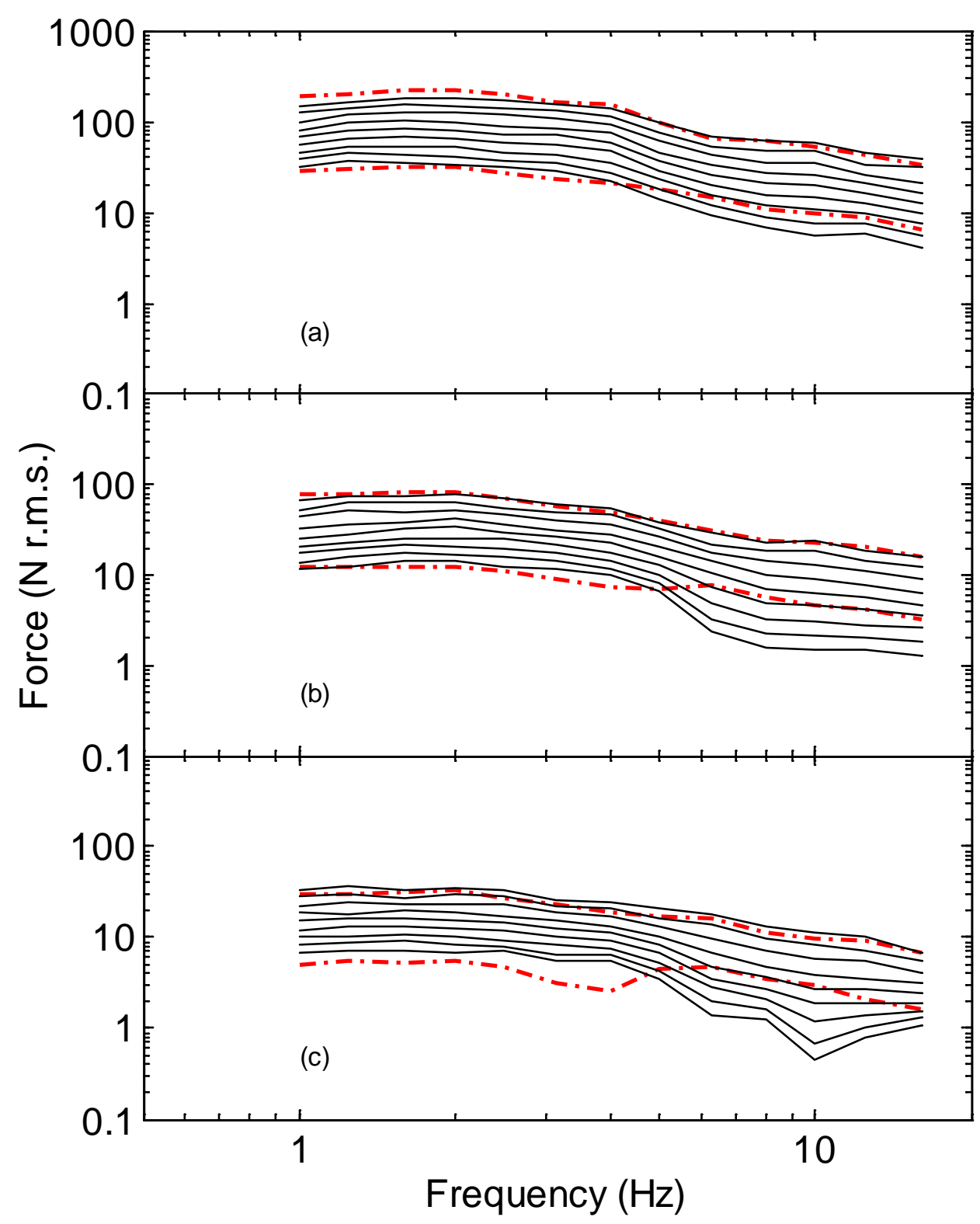

Figure 6 Median force equivalent comfort contours. Contours are shown for subjective magnitudes, $\psi$, of $40,50,63,80,100125,160,200$ and 250 with three magnitudes of $4-\mathrm{Hz}$ reference vibration. (a) $0.8 \mathrm{~ms}^{-2}$ r.m.s. reference; (b) $0.315 \mathrm{~ms}^{-2}$ r.m.s. reference; (c) 0.125 $\mathrm{ms}^{-2}$ r.m.s. reference. The red broken lines show the maximum and minimum force at each frequency (median values over 40 subjects). 


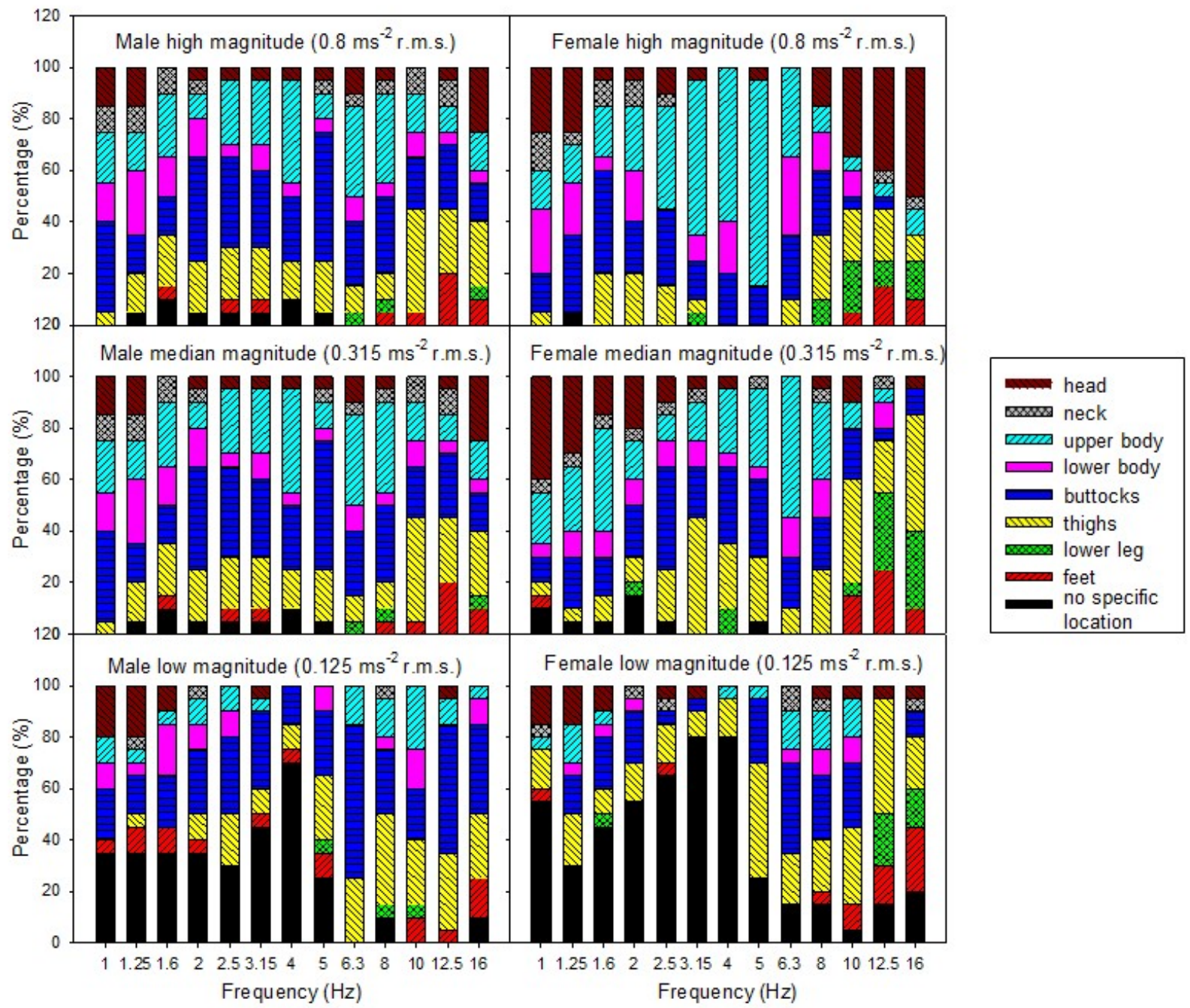

Figure 7 Locations of discomfort arising from exposure to vertical vibration at three different vibration magnitudes for both male and female subjects. 


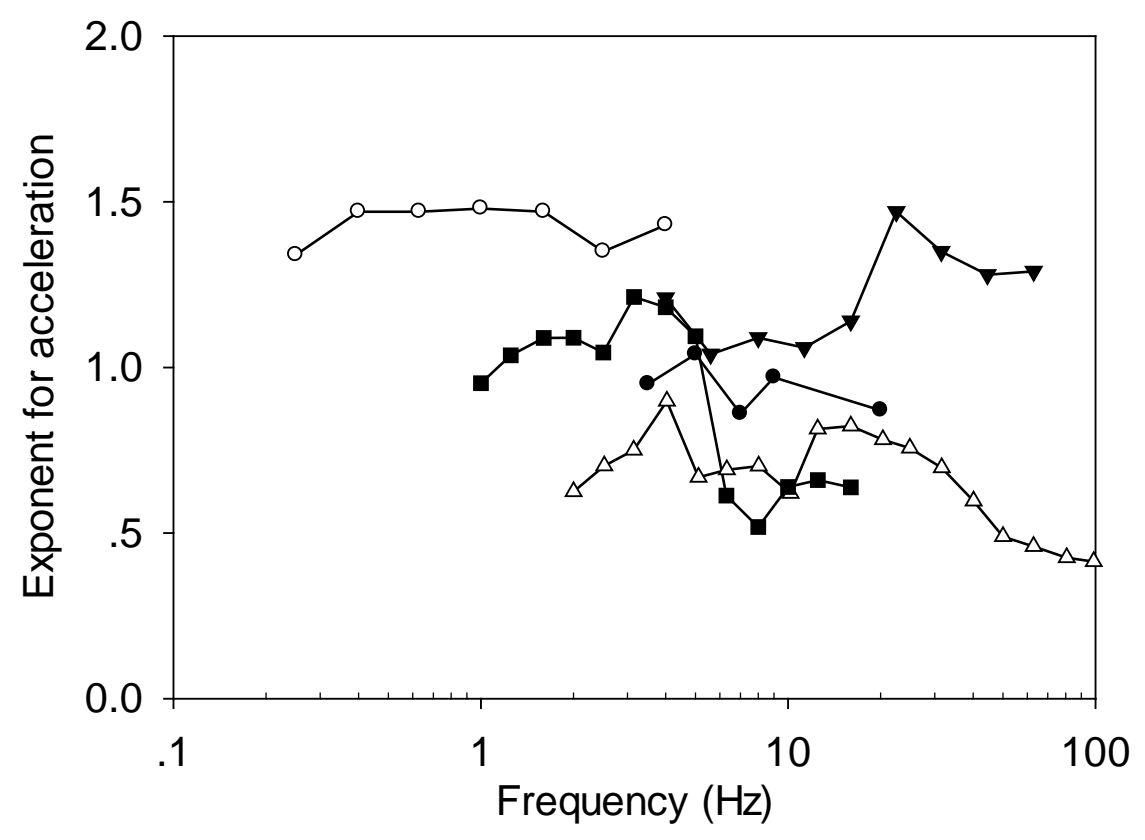

Figure 8 Comparison of the exponent, $n$, from Stevens' power law for acceleration with previous studies (०: Shoenberger and Harris (1971); •: Shoenberger (1975); $\mathbf{\nabla}$ : Howarth and Griffin (1988); $\Delta$ : Morioka and Griffin (2006a); $\mathbf{\square}$ : Present study (medium magnitude session)). 


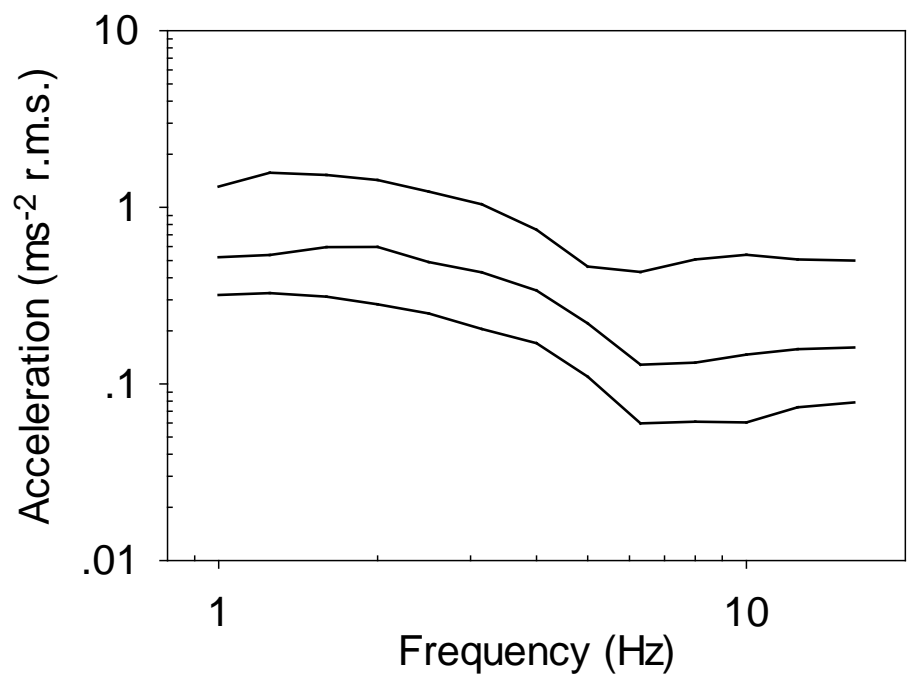

Figure 9 Median equivalent comfort contours obtained for $\psi=100$ with the three magnitudes of reference vibration 


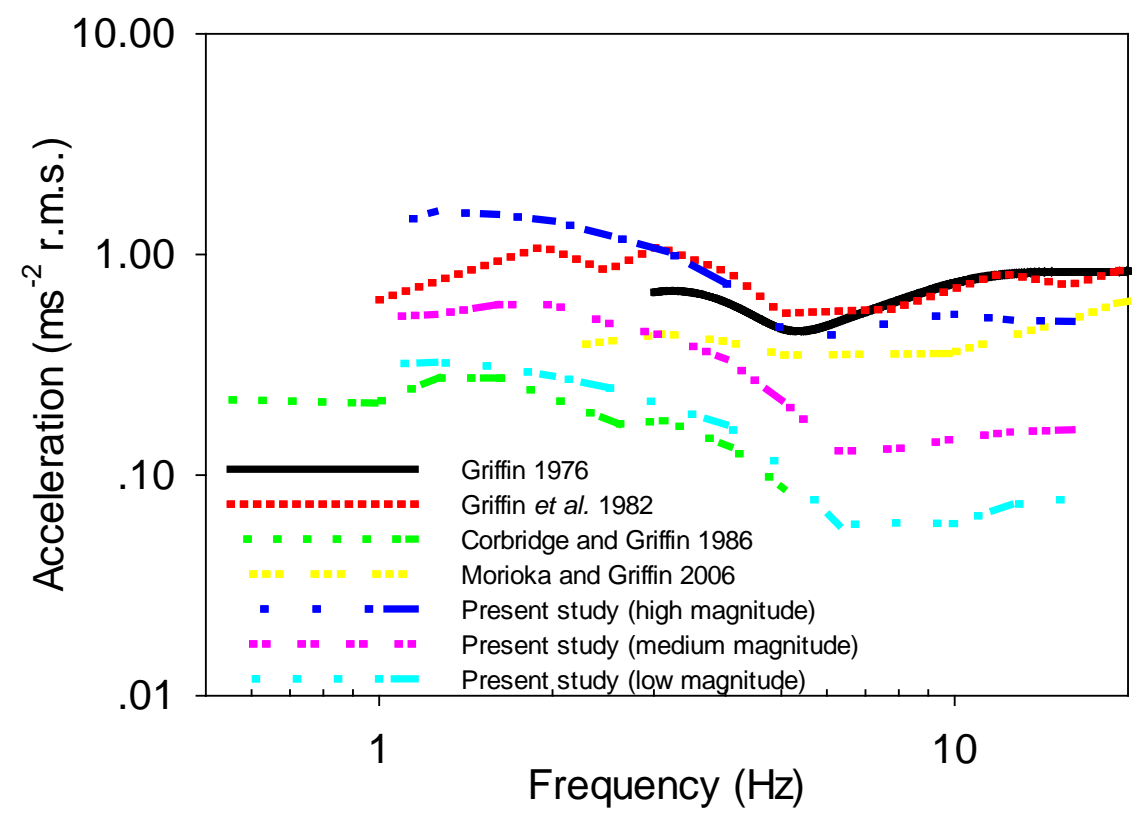

Figure 10 Comparison of equivalent comfort contours from present study with previous studies. 


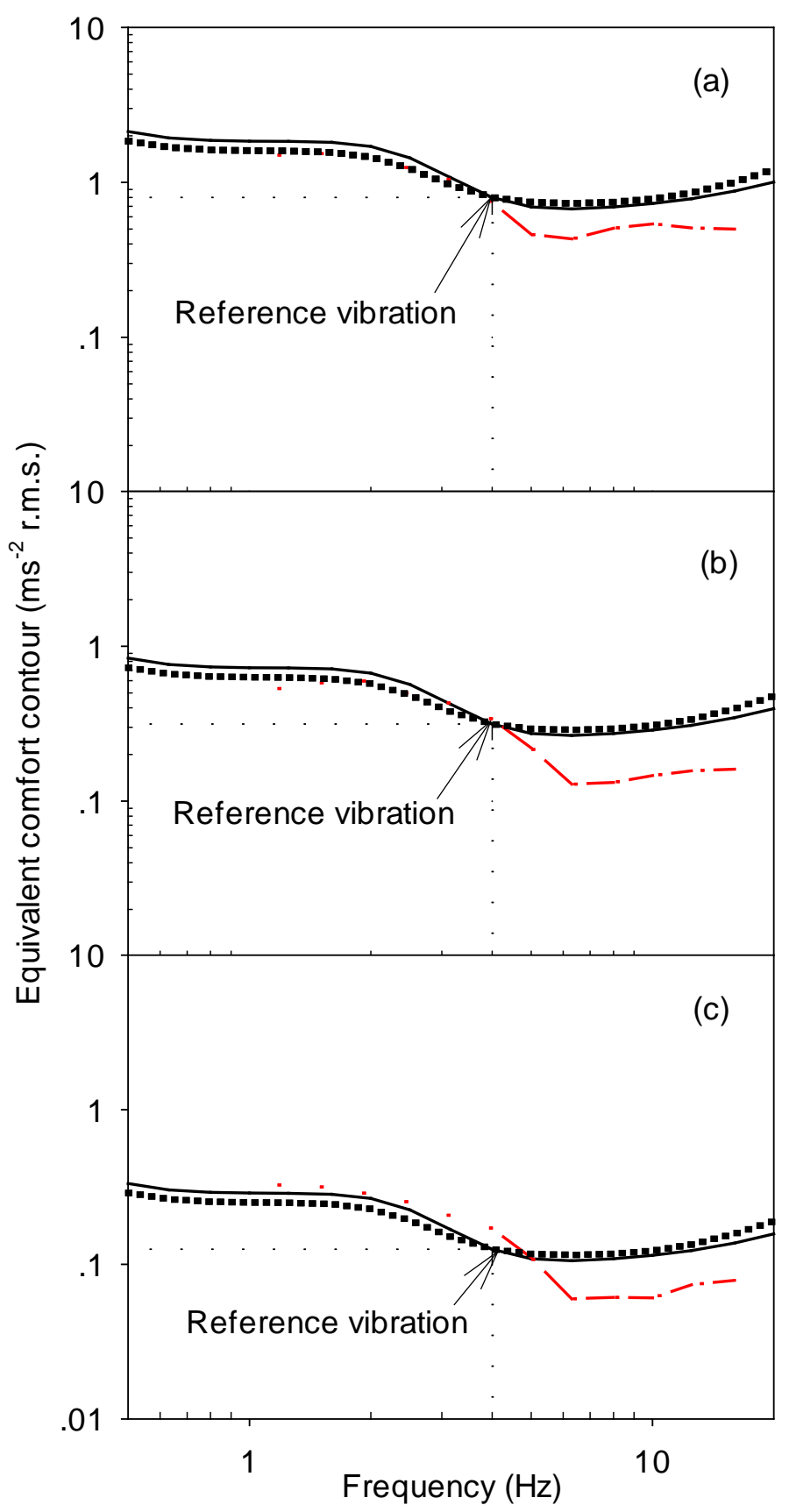

Figure 11 Comparison of the median equivalent comfort contour (for $\psi=100$ ) with the inverted and normalised frequency weightings $W_{\mathrm{b}}$ and $W_{\mathrm{k}}(--$ : equivalent comfort contour; inverted and normalised frequency weighting $W_{\mathrm{b}} ; \cdots \cdot \cdot$ : inverted and normalised frequency weighting $W_{\mathrm{k}}$ ). (a) $0.8 \mathrm{~ms}^{-2}$ r.m.s. reference; (b) $0.315 \mathrm{~ms}^{-2}$ r.m.s. reference; (c) $0.125 \mathrm{~ms}^{-2}$ r.m.s. reference. 
Appendix 1 Individual, median, and inter-quarter ranges of equivalent comfort contours (for $\psi=100$ ) for acceleration: (a) $0.8 \mathrm{~ms}^{-2}$ r.m.s. $4-\mathrm{Hz}$ reference; (b) $0.315 \mathrm{~ms}^{-2}$ r.m.s. $4-\mathrm{Hz}$ reference; (c) $0.125 \mathrm{~ms}^{-2}$ r.m.s. $4-\mathrm{Hz}$ reference.

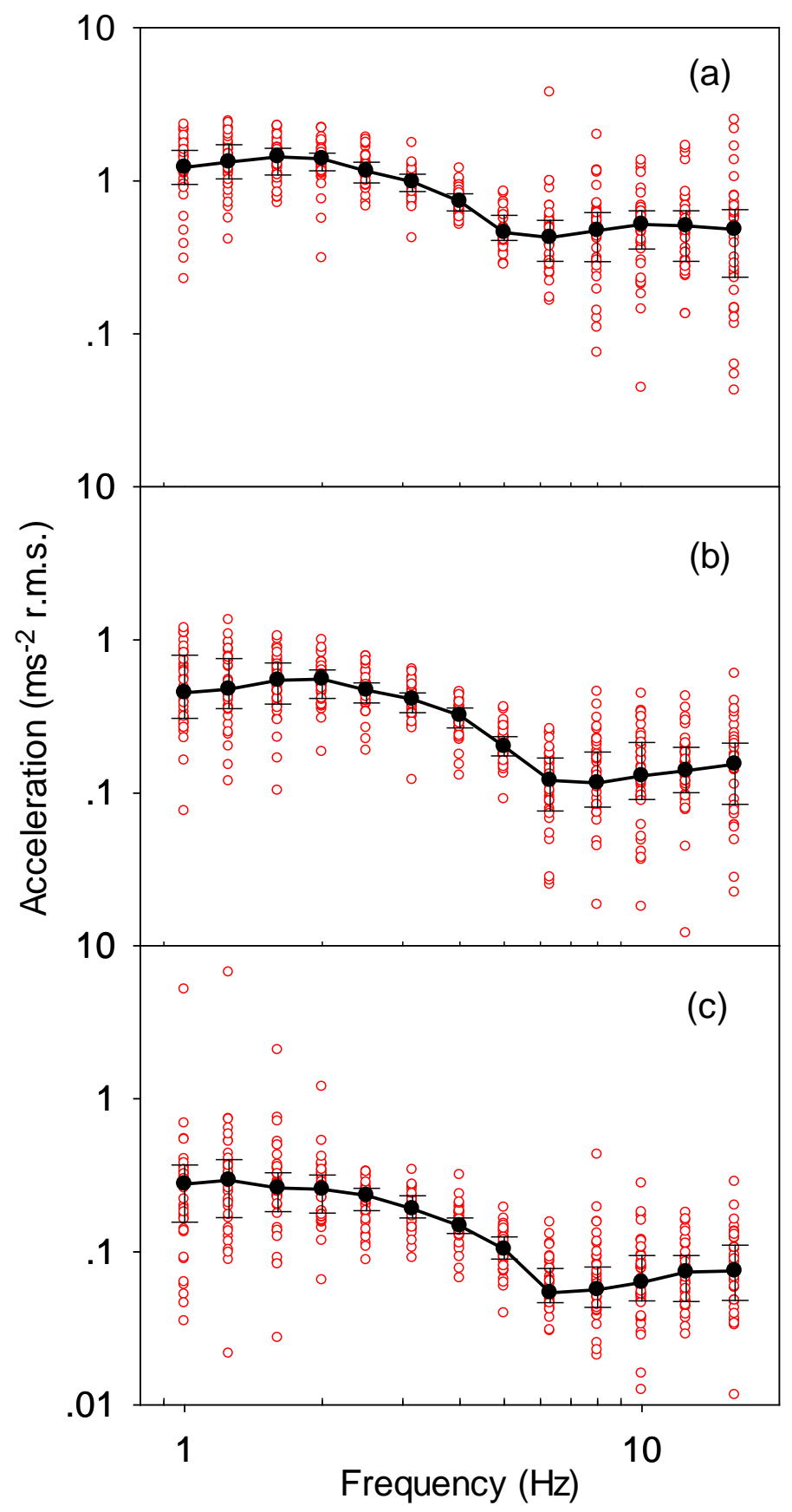


Appendix 2 Individual, median, and inter-quarter ranges of equivalent comfort contours (for $\psi=100$ ) for force (a) $0.8 \mathrm{~ms}^{-2}$ r.m.s. $4-\mathrm{Hz}$ reference; (b) $0.315 \mathrm{~ms}^{-2}$ r.m.s. $4-\mathrm{Hz}$ reference; (c) $0.125 \mathrm{~ms}^{-2}$ r.m.s. $4-\mathrm{Hz}$ reference.

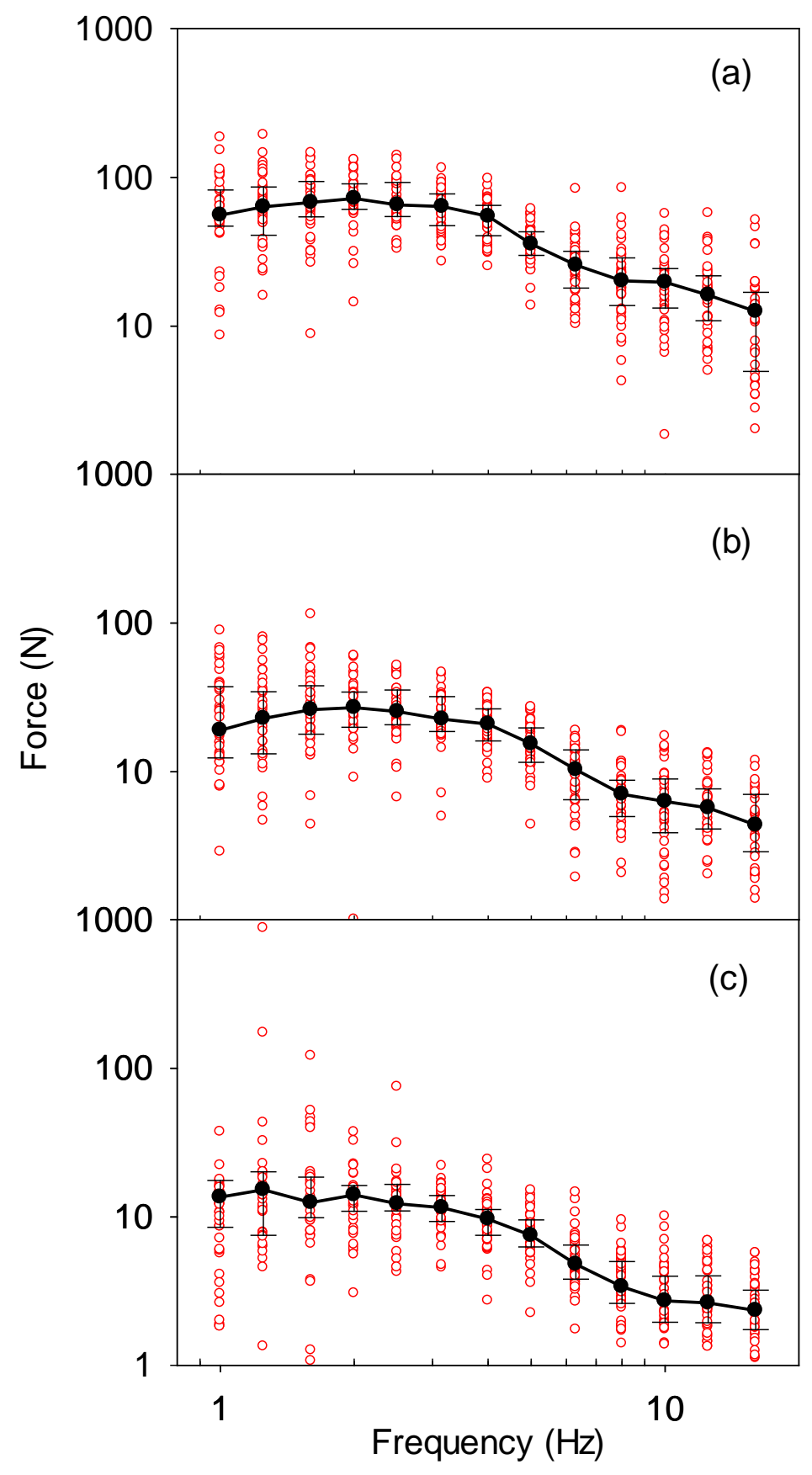

